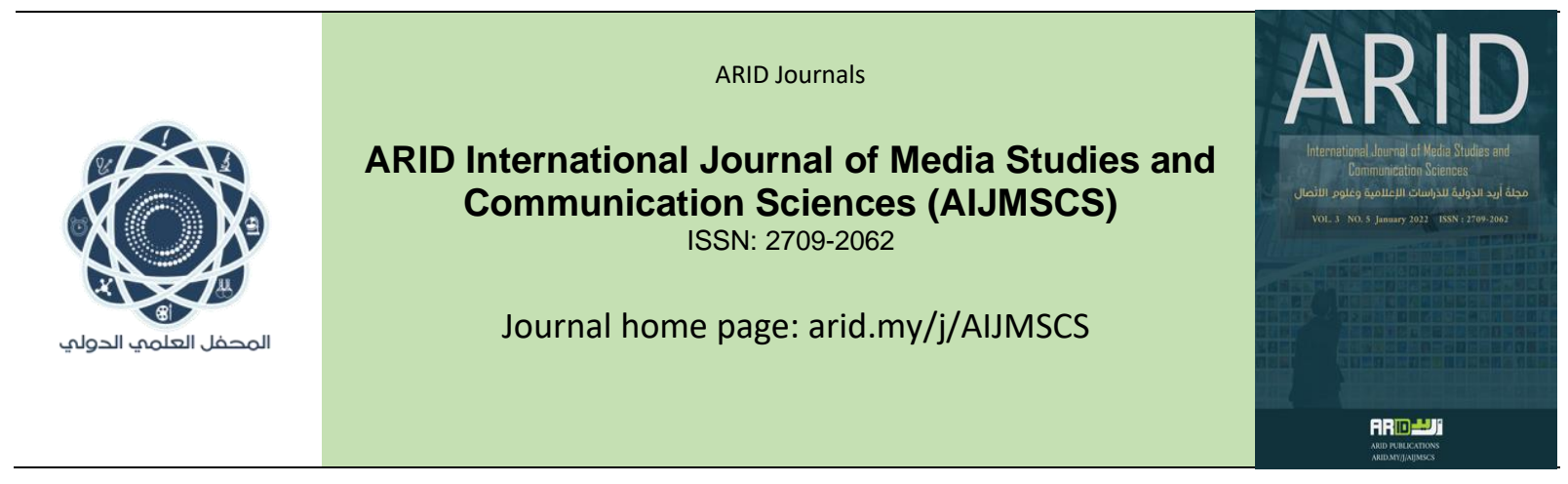

$$
\begin{aligned}
& \text { مَلةُُ أُريد الدَّوليةُ للدراسات الإعلامية وعلوم الإتصال } \\
& \text { المجلد الثالث ،العدد الخامس ،كانون الثاني 2022م }
\end{aligned}
$$

\title{
Recent Trends Of The Researches About The Impact Of The Artificial Intelligence On The Journalism And Television, \\ "A second-level Analytical Study"
}

\author{
Hala Kamal Nofal \\ South Valley University-TV and Radio Department
}

Salwa Ali Ibrahim Elgayyar

Mass Communication and Children Culture,Port Said University

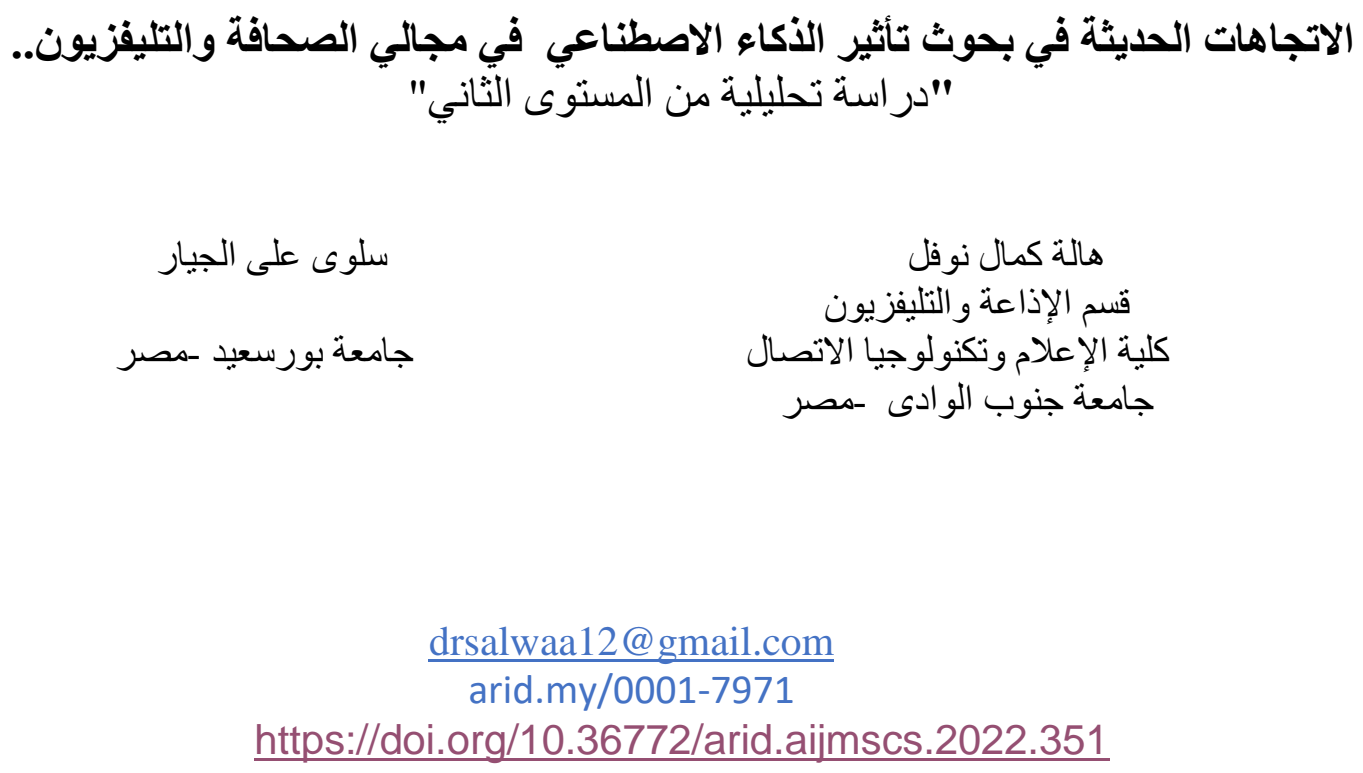




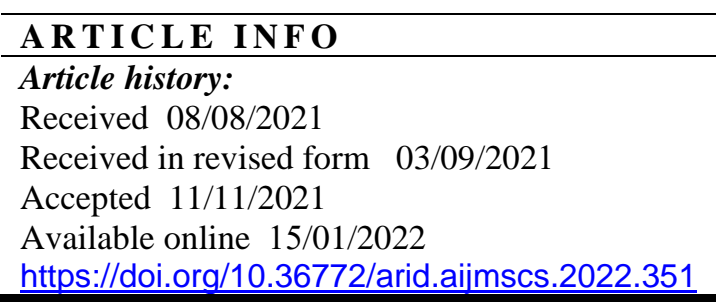

\section{Abstract:}

This study aims to monitor and analyze the recent trends of the researches about the impact of the artificial intelligence on the journalism and television at the level of Arab and foreign studies from different research schools on all over the world in the period from 2017 to 2021.Also, it aims to know the subject fields about the artificial intelligence studies and to know the use of its techniques in journalism and television.

This study is of the descriptive and analytical studies, and it depends on using the analysis style of the second level,According to this, the study depends on the qualitative analysis for the scientific studies related to the field of the artificial intelligence studies and its impact on the journalism and television, The results of this study are the following:

- There is a variety for the recent trends of the researches about the impact of the artificial intelligence on the journalism and television in the period from 2017 to 2021.

- The researches about the impact of artificial intelligence on the journalism are at the top of the interests list with average $(84.2 \%)$, whereas the researches about the impact of artificial intelligence on television are with average (15.8\%), The importance of using the technology of unmanned aerial vehicles (Drones), which allows the journalists to take photos from different angles for the news events such as the volcanic eruptions, war-torn villages and natural disasters.

KEY WORDS: Recent Trends, The Researches About The Impact Of The Artificial Intelligence, The Journalism And Television, A second-level Analytical Study. 


\section{الماخص}

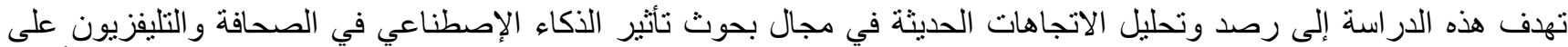

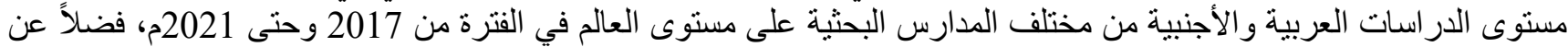

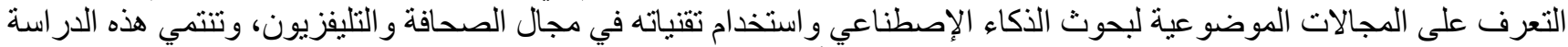

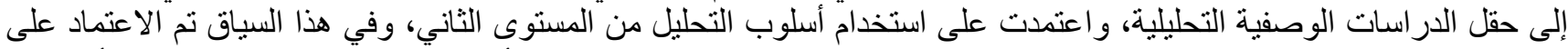

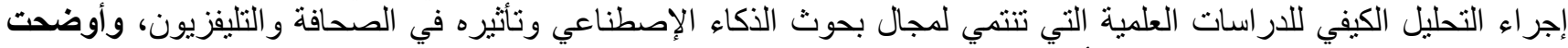

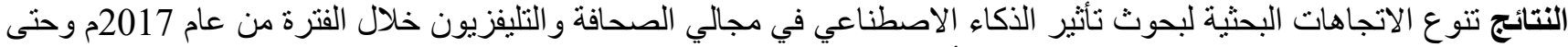

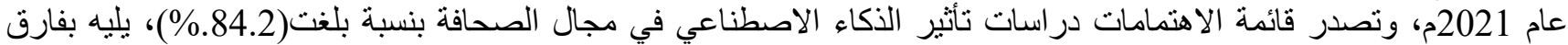

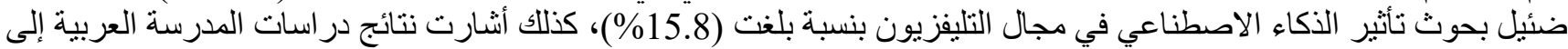

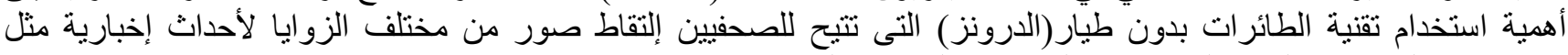

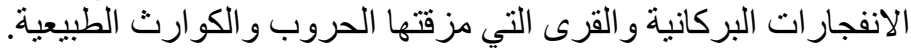

الكلمات المفتاحية:الاتجاهات الحديثة، بحوث تأثير الذكاء الاصطناعي ،الصحافة و التلبفزيون ، در اسة تحليلية من المستوى الثاني 
يعيش العالم المعاصر ثورة تكنولوجية كبيرة في ميدان المعلومات والاتصال، يتضاءل أمامها كل ما تحقق من عدة قرون سابقة، مما كان لها أثرها الكبير على شكل الاتصال ومحتواه وأساليب إنتاجه والمتغيرات المشتركة في عملية الإنتاج، وقد شهدت صناعة الإعلام تغيرات جذرية كبيرة، فمنذ بداية الثورة الصناعية الرابعة؛ التي تعتمد كلياً على الذكاء الاصطناعي، واستخدام الروبوت، الطباعة ثلاثية الأبعاد، منصات البيانات المفتوحة و إنترنت الأشياء، وتحليل البيانات الضخمة، و السرعات الهائلة في شبكات الاتصال، و الهو اتف الذكية بسعتها المهولة، علاوة على الأدوات الجديدة في التصوير و المونتاج، التي سوف تخلق بيانات إعلامية ومعلوماتية بعيدة عن المؤسسات الضخمة، ويلعب فيها الأفر اد دورًا مهمًا بديلًا عن تللك المؤسسات (عبد الظاهر،2019)* 1 .

وقد انتشر الذكاء الاصطناعي بشكل كبير، وبدأ استخدام الريبوت في صناعة المحتوى الإعلامي عبر المنصات الرقمية في إنتاج المحتوى وتطويره، بحيث يقوم به الآلة وليس الإنسان، فالذكاء الاصطناعي عبارة عن تطوير أنظمة حاسوبية قادرة على أداء مهام تتطلب عادة الاستعانة بالذكاء البشري، حيث تقوم فكرته على تحويل الآلة لتقوم بنفس آدوار الجهد البشرى. وفي ظل ما يثهده العالم من تطور ات بفضل ثورة البيانات الضخمة وزيادة قدرات الحوسبة، حقق الذكاء الاصطناعي (AI) انتعانثًا مثيرًا للإعجاب على مدار السنوات القليلة الماضية، و التي أكدت أن الو اقع الافتر اضي و الأنظمة الذكيـة و الأتمتـة سـوف تحل ببطء محل الجوانب المختلفة لكل من الصناعة والتفاعل البشري. كما أن تقنية الطائرات بدون طيار تتيح للصحفيين التقاط لقطات لأحداث إخبارية مثل الانفجارات البركانية والقرى التي مزقتها الحروب والكوارث الطبيعية، نظر اً لأنها يتم تشغلها عن بعد، ويرى الصحفيين أنها وسيلة أكثر أماناً وفعالية من حيث التكلفة لإنتاج الأخبار ولتسجيل الفيديو خاصة في التغطية المعرضة للخطر. ومن دون شك أن تقنيات الذكاء الاصطناعي وتطبيقاته و التطورات الهائلة التي يشهدها العالم ستقود لا محالة إلى ثورة تقنية في قدرة وسائل الإعلام على التأثير ومخاطبة الجمهور وتثكيل الرأي العام (الخولى،2020)، لأن تطبيقاته ستوفر لوسائل الإعلام أدوات أكثر ذكاءً وتقدمًا وسرعة في نقل الخبر إلى المتلقي وتفاعل الأشخاص مع ذللك، وهذا سيشمل وسائل الإعلام المتخصصة، المقروءة والمسموعة و المرئية منها، إضافة إلى وسائل التو اصل الاجتماعي على اختلافها، وهذا يعني أن يكون لثورة الذكاء الاصطناعي تأثثرًا أعمق في صناعة الإعلام والنشر، الأمر الذي سيضاعف من التنافسية للريادة إعلاميًا على 
مستوى الشرق الأوسط والعالم، ومن هنا نجد أن الذكاء الاصطناعي برسم المشهد الإعلامي المستقبلي بناء على أدوار مختلفة تماماً لم تكن موجودة من قبل، و التي أصبح فيه للآلة دور بجانب الإنسان . في ضوء ما سبق تأتي هذه الدر اسة التي تحاول تقديم رؤية نقدية مقارنة من خلال رصد وتحليل الاتجاهات الحديثة في مجال بحوث تأثير الذكاء الاصطناعي في مجالي الصحافة والتليفزيون على مستوى الدراسات العربية والأجنية في الفترة من 2017م وحتى 2021م.

مثكلة الدراسة: تتحدد مشكلة الدراسة الحالية في التساؤل الرئيسي الآتي: ما الاتجاهات الحديثة في بحوث ثأثير الذكاء الاصطناعي في مجالي الصحافة والتليفزيون في كل من الدراسات العربية والأجنبية؟ وما الرؤى المستقبلية لتطوير الأجندة البحثية في هذا المجال؟ أهداف الدراسة: تسعى هذه الدراسة إلى تحقيق مجمو عة من الأهداف، تمثلت في: رصد الدراسات التي تناولت تأثثر الذكاء الاصطناعي في مجالي الصحافة والتليفزيون من مختلف المدارس البحثية على مستوى العالم خلال الفترة من 2017 وحتى 2021م

التعرف على المجالات الموضوعية لبحوث الذكاء الاصطناعي واستخدام تقنياته في مجال الإعلام ومدى تأثيره في مجالي الصحافة و التليفزيون، وذللك على مسنوى الدراسات الأجنبية والعربية عينة الدراسة خلال الفترة من 2017 وحنى

رصد الأطر النظرية التي استندت عليها الدر اسات عينة التحليل، وما قدمته من إضافات على المستوى النظري. التعرف على الأطر المنهجية التي استعانت بها الدراسات عينة التحليل، وما قدمته من إضافات على المسنوى المنهي. السعي لتقديم رؤية مستقبلية واقتر اح أجندة بحثية تسهم في تطويرمجال بحوث الذكاء الاصطناعي ونأثثراته في مجالي الصحافة والتليفزيون، استناداً إلى قاعدة معلومات بشأن التراث العلمي السابق في هذا المجال، والذي يسمح للباحثتين بإجر اء عملية الرصد و التحليل و النقد. 


\section{منهجية الاراسة:}

ينتمي العرض التحليلي الحالي إلى حقل الدراسات الوصفية التحليلية، وتعتمد الدراسة على استخدام أسلوب التحليل من المستوى الثاني "Secondary Analysis وهو أحد أهم الأساليب البحثية التي تستخدم في تحليل المصادر الثانوية، كالدراسات و البحوث و الأدبيات الخاصة بأي مجال من مجالات المعرفة الإنسانية، وفي هذا السياق تم الاعتماد على إجراء التحليل الكيفي للار اسات العلمية الني تنتمي لمجال بحوث الذكاء الاصطناعي وتأثيره في مجالي الصحافة والتليفزيون و المنشورة في الإطار الزمني من عام 2017م وحتى عام 2021م، ويسمح هذا الإطار برصد وتحليل الاتجاهات البحثية المختلفة والتطور الذي يحدث في مجال الذكاء الاصطناعي و علاقته بمجالي الصحافة و التليفزيون ورؤى و أفكار الباحثين في هذا المجال.

\section{مجتمع وعينة الاراسة:}

يتمثل مجتمع الدراسة الحالية في البحوث العربية والأجنية التي تناولت تطبيقات الذكاء الاصطناعي ومدى استخدامها وتأثيراتها في مجال الصحافة والتليفزيون، وفيما يتعلق بعينة الدراسة، تم القيام بوصف وتحليل لبحوث ودراسات الذكاء الاصطناعي في مجالي الصحافة و التليفزيون العربية والأجنية خلال الفترة من عام 2017م وحتى 2021م، حيث بلغ إجمالي عدد البحوث التي تم الاستعانة بها في العرض التحليلي (38) دراسة، منهم (11) دراسة عربية و(27) دراسة أجنيبة، كما استعانت الباحثنان في جمع الدراسات العربية و الأجنبية عينة الدراسة بقو اعد البيانات العربية والأجنبية على شبكة الإنترنت وعدد من المو اقع الأكاديمية، ومو اقع الهجلات العلمية العربية وتمثلت في المصادر الآتية:

\section{جدول(1): قواعد البيانات التي اعتمدت عليها الاراسة}

\begin{tabular}{|c|c|}
\hline 8- Willey Online Library & 1- موقع اتحاد مكتبات الجامعات المصرية. \\
\hline 9- Web of Science & 2- بنك المعرفة المصرى. . \\
\hline 10- Sage. & 3- مو اقع الدوريات المصرية عبر موقع بنك المعرفة المصرى. \\
\hline 11-Eric & 4- دار الَنظومة. \\
\hline 12-IEEE Xplor. & 5- Science Direct \\
\hline 13- Emerlad Insight & 6- Reserch Gate \\
\hline 14- Google Scholar & 7- Questia \\
\hline
\end{tabular}


جدول(2): يوضح توصيف العينة لبحوث تأثير الأكاء الاصطناعي في مجالي الصحافة والتليفزيون في الاراسات العربية والأجنبية

\begin{tabular}{|c|c|c|c|c|c|c|c|c|c|c|}
\hline \multicolumn{2}{|c|}{ الإجمالى } & \multicolumn{2}{|c|}{ الأسراسيوية } & \multicolumn{2}{|c|}{ الألمر اسكية } & \multicolumn{2}{|c|}{ الأوراسيات } & \multicolumn{2}{|c|}{ الألعربية } & \multirow{2}{*}{ 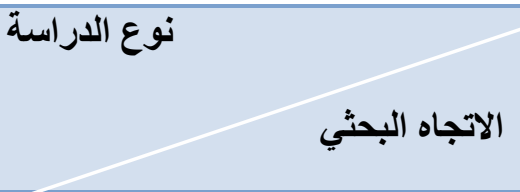 } \\
\hline$\%$ & ك & $\%$ & ك & $\%$ & ك & $\%$ & ك & $\%$ & ك & \\
\hline 84.2 & 32 & 9.4 & 3 & 15.6 & 5 & 40.6 & 13 & 34.4 & 11 & تأثير الأكاء الاصطناعي في مجال \\
\hline 15.8 & 6 & - & - & 33.3 & 2 & 66.7 & 4 & - & - & تأتثير الذكاء الاصطناعي في مجال \\
\hline 100 & 38 & 7.9 & 3 & 18.4 & 7 & 44.7 & 17 & 28.9 & 11 & \\
\hline
\end{tabular}

يتضح من الجدول السابق: تنوع الاتجاهات البحثية لبحوث نأثير الذكاء الاصطناعي في مجالي الصحافة والتليفزيون خلال الفترة من عام 2017م وحتى عام 2021م، وتصدر قائمة الاهتمامات در اسات تأثير الذكاء الاصطناعي في مجال الصحافة بنسبة بلغت(84.2\%)، يليه بفارق ضئيل جداً بحوث تأثير الذكاء الاصطناعي في مجال التليفزيون بنسبة بلغت (15.8\%). • كما يشير الجدول إلى أن الدراسات الأوربية جاءت في الترتيب الأول بنسبة (44.7\%)، يليها في الترتيب الثاني الدراسات العربية بنسبة بلغت (28.9\%) ،وتأتي الدر اسات الأمريكية في الترتيب الثالث بنسبة (18.4\%)، ثم الدراسات الأسيوية بنسبة بلغت (\% (\%).

• أثنارت نتائج الجدول أيضاً إلى تفوق المدرسة العربية على المدرسة الأمريكية والأسيوية في دراسات بحوث تأثثر الذكاء الاصطناعي في مجالي الصحافة و التليفزيون بنحو (11) در اسة في مقابل (7) در اسات للمدرسة الأمريكية، و(3) دراسات فقط للمدرسة الأسيوية مما يعطى مؤشراً هاماً على اهتمام الدراسات العربية بدر اسة هذا الموضوع المهم، على الرغم مما أثشارت إليه النتائج فيما بعد من ندرة استخدام تطبيقات الذكاء الصناعي في العديد من المؤسسات الاعلامية وخاصة الصحفية 
• مراجعة الاتجاهات الحديثة لبحوث والاراسات العربية والأجنبية التي تناولت تأثير الأكاء الاصطناعي في مجالي الصحافة والتليفزيون: - و

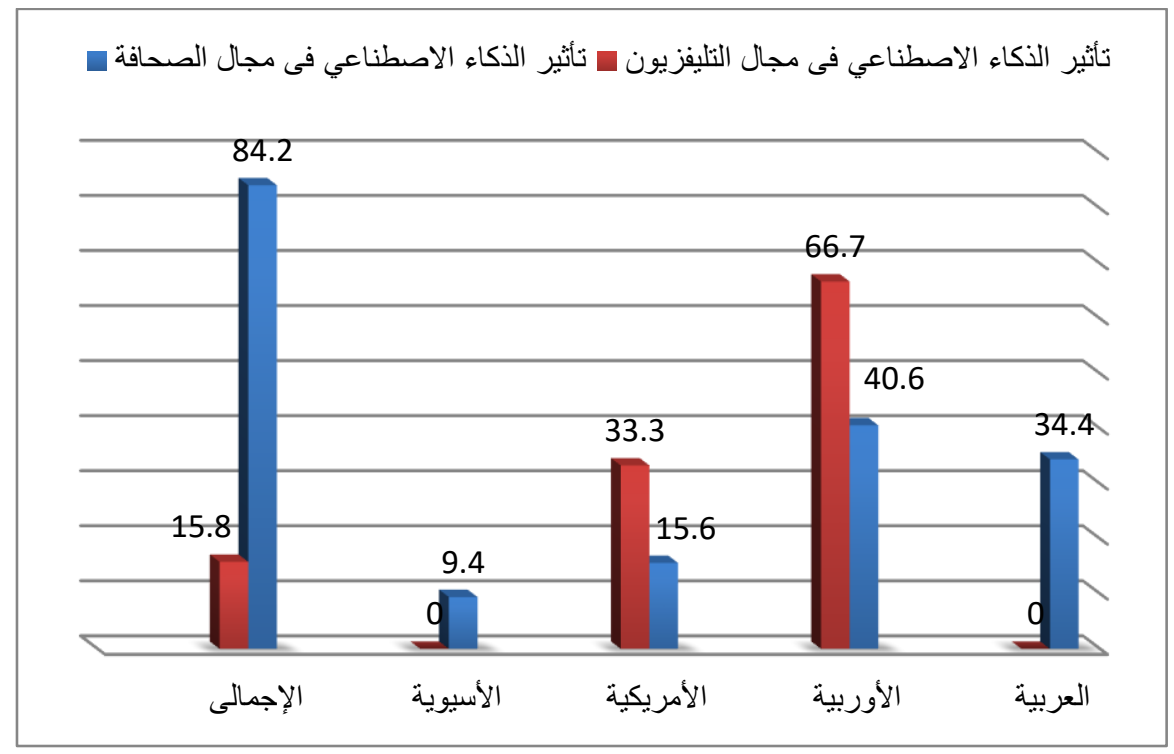

شكل(1): يوضح الاتجاهات الحديثة لبحوث تأثير الأكاء الاصطناعي في مجالي الصحافة والتليفزيون في الدراسات العربية والأجنبية. وسيتم تناول تلكك الاتجاهات البحثية السابقة من خلال محوريين رئيسين كما يلي:1- المحورالأول: استعر اض الدر اسات التي تناولت تأثثر الذكاء الاصطناعي في مجال الصحافة. 2- المحور الثاني: استعر اض الدر اسات التي تناولت تأثثر الذكاء الاصطناعي في مجال التليفزيون. المحور الأول: استعراض الاراسات التي تناولت تأثير الأكاء الاصطناعي في مجال الصحافة:رصدت الباحثة في إطار هذا المحور (32) دراسة عربية وأجنبية، استأثرت الدراسات الأجنبية منها بـ(21) دراسة، بنسبة بلغت (65.6\%)، كان منها (13) در اسة أوربية و(5) أمريكية و(3) أسيوية، وذلك في مقابل (11) دراسات عربية بنسبة (34.4) (34)، وتم تقسيم الدر اسات إلى محوريين فرعيين. يضم المحور الأول: الدراسات التي تناولت تأثير استخدام الذكاء الاصطناعي في العمل الصحفي، ويتناول الثانى: اتجاهات القائمين بالاتصال نحو استخدام تقنيات الذكاء الاصطناعي بالمؤسسات الصحفية، وفيما يلي عرض تفصيلي لكل محور من تلك المحاور:(أ) المحور الفرعى الأول: الاراسات التى تناولت تأثثير استخدام الأكاء الاصطناعى في العمل الصحفى:فعلى مستوى استخدام الذكاء الاصطناعي في العمل الصحفي سعت الدراسة العربية (بدوى، 2021) للتعرف على كيفية تطبيق صحافة الروبوت وآليات إنتاجها في موقع القاهرة 24 الإخباري، بإعتباره أول موقع مصري يطبق هذا النموذج من 
تقنيات الذكاء الاصطناعي، وقد اعتمدت على المنهج المسحي واستمارة الاستبانة في جمع البيانات من عينة عشوائية من الصحفيين بالموقع، واستخدمت النظرية الموحدة لقبول واستخدام التكنولوجيا، وتوصلت إلى أهمية تطبيق صحافة الريبوت في المو اقع الصحفية المصرية لقدرتها على تقديم محتوى صحفي أكثر تمييز أ وأكثر مصداقية لدى الجمهور. و على نحو آخر جاءت دراسة (عبد المعطى،2021) حول دور الأكاء الاصطناعي في مجال الصحافة أثناء الأزمات والكوراث، لتؤكد أهمية ابتكار طرق تقنية وتكنولوجية جديدة لتغطية الكوارث والأزمات باستخدام الذكاء الاصطناعي للحفاظ على حياة الصحفيين وتتتمسي هذه الدراسة إلى الدراسات الوصفية والاستشر افية، وتمثل عينة الدراسة في المختصين بمجال الذكاء الاصطناعي، وقد استعانت الباحثة بأداة المقابلة المتعقة، وتوصلت الدراسة إلى أن استخدام الصحفي لتقنيات الذكاء الاصطناعي تعمل على تأمين حياته، ومر اقبة الأماكن التي يصعب الوصول إليها.

كذلك هدفت الاراسة العربية (منصور،2021) إلى الكثف عن مستقبل الصحافة المصرية في ظل تقتيات الأكاء الاصطناعي خلال العقد من (2030-2021)، واستندت في بناء متغير اتها وتفسير نتائجها على السيناريو هات المنوقعة لمستقبل الصحافة في ظل تقنيات الذكاء الاصطناعي ومدخل نظم المعلومات، واعتمدت على المنهج الوصفي و أداتي الاستبانة و المقابلات المتعمقة، وذلك بالتطبيق على عينة قو امها 50 صحفيًا، وتوصلت إلى أن استخدام صحافة الذكاء الاصطناعي في الصحف مستقبلاً لمو اجهة انخفاض قارئية الصحف ورفع كفاءة العمل.

بينما جاءت الدراسة الأسيوية (Biswal \& Gouda, 2020) للتعرف على تأثيراستخدام الأكاء الاصطناعي في وكالة أنباءXinhua الصينية على العمل الصحفي، وما هي التحديات التي تعيق توظيف تللك التكنولوجيا في الوكالة، وقد اعتمد الباحث في در استه على المنهج المسحي، حيث قام باختيار عينة عشو ائية قوامها 25 صحفيًا من وكالة أنباء Xinhua الصينية، وباستخدام المقابلات المتعمقة، أشارت النتائج أن الذكاء الاصطناعي أسهم بشكل كبير في العمل الصحفي، خاصة فيما يتعلق بجمع وتحرير المعلومات والبيانات الذي يحدث إلكترونيًا ودون التدخل المباثر من قبل المحريين، مما وفر الكثير من الوقت و الجهد اللازم في العمل الصحفي للوكالة، كما توصلت أن الذكاء الاصطناعي مكن وكالة Xinhua من نطوير أشكال جديدة من عرض القصص الخبرية ومنها الاعتماد على ما يعرف بالو اقع الافتراضي Virtual Realityحيث يتم عرض الأخبار في صورة تشبه الأفلام ثلاثية الأبعاد تجعل المشاهد يعيش تجربة الخبر كأنها واقع وهو عضو شارك فيه.

و أضافت دراسة (Leavy, 2020) بعنوان: استخدام الذكاء الصناعي في العمل الصحفي وعلاقته بالتحيز في تغطية الأخبار المتعلقة بالمرشحين السياسيين"، والتي حاولت التعرف على أسباب التحيز في الأخبار المتعلقة بالمرشحين السياسيين الإناث في دولة إيرلندا، وهل هنالك تأثير سلبي على سلوكيات ووعي الجمهور فيما يتعلق بالمرشحين، وقد اعتمد الباحث في دراسته 
على المنهج المسحي، حيث قام بإجر اء دراسة تحليلية طويلة قام من خلالها بتحليل عينة قوامها 469 من أصل 47981 مقالاً متعلقًا بالمرشحين، والتي نم نشرها في صحيفتي The Independent \& The sunday، وقد أشارت نتائج الدراسة أن البر امج التي يتم الاستعانة بها في الذكاء الاصطناعي في العمل الصحفي تعتمد على لغة اللوغاريتمات، حيث أنها اعتمدت في تحليلها على المقالات و الأخبار الصحفية السابقة والتي نشرت على المرشحات والتي كانت في الأغلب تتجه نحو التحيز ضدهن.

ومن الدراسات العربية خاصة المصرية تناولت دراسة (عبد المعط،2020)، عن دور الذكاء الاصطناعي في تطوير الصحافة الإكترونية، في محاولة للتعرف على مستقبل هذه التقنية في مجال الصحافة وكيفية الاستفادة منها ومحاولة تطوير ها، ورصد طرق توعية المؤسسات الصحفية باستخدام تقنيات الذكاء الاصطناعي، وقد أثنتت نتائج الدراسة أن صحافة الذكاء الاصطناعي حقبة جديدة من الإعلام تسعى من خلالها وسائل الإعلام إلى توظيف تقنيات الذكاء الاصطناعي و الثورة الصناعية الر ابعة على أكمل وجه في جميع مر احل صناعة الإعلام وصناعة الخبر، والتي ستكون أسرع عشرات المرات من وسائل التو اصل الاجتماعي، و أكثر دقة وتفصيلاً في نقل الأحداث، و أثنارت إلى أهمية توظيف الروبوت في التحرير و التصوير، وفي تغطية الأحداث بالأماكن الأكثر خطورة ويصعب على الإنسان الوصول إليها مثل مناطق الحروب والحرائق وقاع البحار و الفضاء وفوق ناطحات السحاب.

كذلك برزت در اسة (نوفل،2020) حول صحافة الذكاء الاصطناعى واستخدامات الدرونز في الإعلام أنشارت الدراسة أن تقنية الطائر ات بدون طيار تتيح للصحفيين التقاط لقطات لأحداث إخبارية مثل الانفجار ات البركانية والقرى التى مزقتها الحروب والكوارث الطبيعية، نظر آ لأنها يتم تشغلها عن بعد، ويرى الصحفيين أنها وسيلة أكثر أماناً وفعالية من حيث التكلفة لإنتاج الأخبار ولتسجيل الفيديو خاصة في التغطية المعرضة للخطر، كذلك استخدام صحافة الطائرات بدون طيار، يمكن الحصول على صور جوية بتنسيق المو اقف التى يصعب تغطينها بمنظور وسر عة التنقل، وسلامة أكبر للصحفيين، وبتكلفة أقل، كما أشارت الدر اسة إلى الظروف والأسباب الكامنة وراء بطء اندماجهم في غرف الأخبار مثل تكلفة الاستثمار في التكنولوجيا بعمر قصير بسبب تطور ها المستمر و التعقيد الفني والإداري، فضلا عن عدم وجود صحفيين متخصصين وقو انين مقيدة. وفي نفس السياق برزت در اسة (بدوى، 2020) التي استهدفت مستقبل الوظيفة الإخبارية للصحافة الإكترونية في ظل تعدد منصات الإعلام الرقمي، دراسة مستقبلية في الفترة من 2018 وحتى 2028، في ضوء استخدام تقتيات الذكاء الاصطناعي رصد و اقع الوظيفة الإخبارية للصحف و المو اقع الإلكترونية، و التعرف على طبيعة الوظيفة الإخبارية في العصر الرقمي، وآلياتها وممارساتها الجديدة، والتحديات التي تواجهها، والتعرف على آراء الخبراء من المهنيين العاملين بالمجال 
الإعلامي و الأكاديميين وتصور اتهم حول مستقبل الوظيفة الإخبارية في البيئة الرقية في ظل تعدد المنصات الإعلامية، وتتنمي هذه الدر اسة إلى نمط الدر اسات المستقبلة الاستكثافية أو الاستطلاعية، حيث تحاول الكثف عن المستقبل المتوقع أو المحتمل وقد استعانت الدراسة بمنهج المسح الإعلامي، والمنهج المقارن، كما تم إجراء الدراسة التطبيقية على عينة قو امها (100) مفردة من الخبراء المهنيين العاملين بالمجال الإعلامي والأكاديميين واعتمدت الدراسة في إطار ها النظري على مدخل تحليل النظم، وقد توصلت الدر اسة في ضوء استخدام تقنيات الذكاء الاصطناعي أن الصحافة الرقمية المصرية عند مقارنتها بمثيلاتها في أوروبا أو أمريكا أو أسيا، يتبين أنها تتحسس خطاها نحو التطوير لكن يعيبها البطء الثنديد، ويجب عليها إذا ما أرادت أن تحجز لها مقعداً في المستقبل أن تأخذ بالآتي: التبني الجاد والحققي لأدوات الذكاء الاصطناعي في التحرير الرقمي، وتدريب الصحفيين على العمل وفق ممارسات البيئة الرقمية.

وباستخدام دراسة الحالة سعت الدراسة الأوربية (Braghieri,2019) لرصد تأثير استخدام التكنولوجيا الحديثة و الذكاء الاصطناعي على العمل الصحفي وعلى أداء الصحفيين، إلى التعرف على أهمية الذكاء الاصطناعي في جمع ونشر المعلومات، حيث قام الباحث باختيار مؤسسة صحفية Daily Express البريطانية ودر استها لمدة طويلة Long- term لمعرفة مدى تأثثر الذكاء الاصطناعي على أداء المهام الصحفية، واستخدم الباحث استمارة الاستقصاء للحصول على المعلومات من الصحفيين من المؤسسة، وقد أشارت نتائج الدراسة أن الذكاء الاصطناعي أسهم بشكل كبير في تدفق وتوزيع ونشر المعلومات التي تقوم المؤسسة الصحفية بإنتاجها، كذلك أكدت على أهية توظيف الذكاء الاصطناعي في نشر الأخبار من خلال النطبيق الذي تصدره صحيفة Daily Express عن طريق استخدام قارئ الأخبار الاصطناعي Artificial Anchorman و الذي يقوم بدور المذيع للأخر إلى الجمهور على غرار قارئ النشرة الموجود في الر اديو أو التليفزيون. وجاءت دراسة (Ufarte Ruiz \& Manfredi Sánchez, 2019) تتناول دراسة حالة على شركة NarrativaInteligencia Artificial العمليات التنظية و إجر اءات العمل وجودة المعلومات المنتجة بو اسطة تلك الثركة والتي تعد الثركة الوحيدة في إسبانيا التى تكتب نصوصًا صحفية باستخدام برنامج يسمى Gabriele (نوع من أنواع الذكاء الاصطناعي)، و الذى يستخدم لتوزيع الأخبار لوسائل الإعلام المختلفة، وباستخدام منهج المسح أجريت در اسة على عينة من العاملين بالثركة وعينة من الصحفيين وكذلك استخدام الملاحظة لتتبع كيفية العمل بالشركة، وشملت عينة الخبر اء بالثركة أربعة خبر اء" تم إجر اء مقابلات متعدقة معهم بينما اشتملت عينة الصحفيين 145 صحفيًا لتقييم جودة النصوص المنتجة، وأظهرت النتائج أن البرنامج أدى إلى زيادة الإنتاجية الصحفية من خلال الإنتاج الآلي Automatic و الدقيق و السريع للنصوص الصحفية والتي وصلت إلى 20 ألف نص 
في الأسبوع الو احد، وبتتبع النصوص التي تم إنتاجها لوحظ أنها لا تختلف كثيرًا من حيث الأسلوب و التكوين و البناء عن الثكل التقليدي بالصحافة التقليدية.

كما سعت الدر اسة الأمريكية (Stray, 2019) للتعرف على مدى توظيف واستخدام تقتيات الذكاء الاصطناعي في مجال الصحافة الاستقصائية، وذلك من خلال التعرف على تقارير الصحافة الاستقصائية ومشكلاتها التي يمكن حلها باستخدام تقنيات الذكاء الاصطناعي وتحليل عدد من الحالات، وتوصلت الدراسة إلى أن استخدامات للذكاء الاصطناعي في الصحافة الاستقصائية منو اضع للغاية، ولوحظ أنه لم يكن بالإمكان إنتاج تللك القصص بدون تلك الآليات، فلقد أصبحت مطلوبة للغاية أكثر لأن عدم استخدامها يؤدي إلى الاعتماد على عدد كبير من العمالة اليدوية. ومن منظور آخر سعت دراسة (Tatalovic, 2018)إلى الوقوف على مدى الاعتماد على تقنيات الأكاء الاصطناعي في الصحافة العلمية من خلال تتخيص الدر اسات والأبحاث العلمية ووضعها في شكل تقارير صحفية عن طريق استخدام تقنيات الأتمنة، كما حدث ذلك في بعض الصحف فيما يتعلق بالموضو عات الرياضية و السياسية والاقتصادية، حيث توصلت الدراسة إلى أن الصحافة العلمية لم تستفد حتى الأن من تقنيات الذكاء الاصطناعي مثلما حدث في الصحافة الرياضية، وأن المحررين العاملين في مجال الصحافة العلمية لا يزالون غير مدركين لأهمية استخدام تقنيات الذكاء الاصطناعي في إعداد التقارير الخاصة بالصحافة العلمية. ومن الاراسات العربية أيضا جاءت دراسة (أحمد، 2018)، حول مستقبل الصحافة الإلكترونية في إطار تقنيات الواقع الإفتر اضي، وقد بحثت تلك الدر اسة في التطور ات المتعلقة بالصحافة وتحديداً صحافة الو اقع الافتر اضي، وكيفية تطبيق تقنيات الو اقع الافتراضي في مجالات القصص الصحفية، وتحاول تللك الدراسة تقديم مفهوم جديد عن الصحافة الغامرة وأهميتها وتطور ها كل ذلك من خلال در اسة تحليلية من المستوى الثناني من خلال التحليل الكيفي لنتائج البحوث من و اقع التراث العلمي و المقالات وكل ما كتب حول الصحافة الغامرة وإدر اجه ضمن مفاهيم الصحافة، و أكدت الدراسة على احتياج الصحفيين لفهم أفضل للمفاهيم الأسياسية للانغماس وقدرات تقنيات الغمر الشائعة وحدودها، ومعرفة الأساسيات التي تحدد الاحتر افية والتفوق الصحفي.

وعن استخدام الأكاء الاصطناعي في الصحافة الآلية، جاعت در اسـة (Schapals \& Porlezza, 2020)بعـوان مســاعدة أم مقاومـةت؟ تقيـيم للصـحافة المميكــة ومفـاهيم الـدور الصـحفي، وباسـتخدام نمـوذج Hanitzsch و Vos لـلأدوار الصـحفية وتفسير Deuzeللصحافة باعتبار ها أيدلويجية كإطارين نظريين للار اسة ركزت الدراسة على التعرف على المـاخل الوصفية للصحافة الآلية Automated Journalism و التعرف إلى أي مدى تغيرت الأدوار الصحفية أو تطورت نتيجة استخدام الذكاء 
الاصطناعي، واختيرت غرفتان من غرف الأخبار وعقد مقابلات مـع العـاملين بها وبلـغن عدد المبحـوثين 73 مـن المحررين و الصحفيين، وأكلدت نتائج الدراسة أن كافة المبحوثين ما عدا مبحوثناً واحداً يـرون أنـه لا يوجد احتبـاج للتغيير مـن أدوار هـ الصحفية للتواؤم مع تكنولوجيا الذكاء الاصطناعي في المستقبل، كما أعربو ا عن تمسكمه بأدو ارهم، وأن الصحافة لديهم بمثابة الأيديولوجية مما يثـجعهم لمو اجهة الميكنـة Automation، كمـا يـرون أن المسـتحدثات التكنولوجيـة لا تمثنل لهم تهديدًا ولا يقلقون عن احتمالية أن تحل الآلة محلهم في المستقبل، وأن تلك التكنولوجيـات هي أدوات مسـاعدة للصحفيين في آداء عملهم الإخباري اليومي وتمكنهم من آداء مهام صعبة عليهح.

واتفقت معها دراسة (Ali \& Hassoun, 2019)، بعنوان:"الأكاء الاصطناعي والصحافة الآلية: التحديات المعاصرة والفرص الجديدة، في وصف الوضع الراهن للتكنولوجيا وذلك بالتركيز على دور الذكاء الاصطناعي في تغيير الممارسة الصحفية، والوقوف على الآثار المحتملة على مستقبل الصحفيين جراء توظيف تطبيقات الذكاء الاصطناعي، واستنباط التحديات الأخلاقية والمهنية التي قد تزعج ممارسات مهنة الصحافة جراء ظهور هذه التقنيات الحديثة، وفي هذا الإطار تقوم الدر اسة الحالية بمر اجعة منهجية للأدبيات، و الدر اسات السابقة، حيث توصلت الدراسة إلى أن تقنيات الذكاء الاصطناعي تمثل تطورًا كبيرًا في بيئة العمل الصحفي في العصر الرقمي، خاصة في ظل قدرنها على التغلب على المشاكل الأساسية التي تواجه الصحافة المعاصرة ومكافحة الأخبار المزيفة، وتحرير الأخبار، وكذلك تخصيص المحتوى، كما أثنارت أيضًا إلى أن استخدام تطبيقات الذكاء الاصطناعي في الصحافة يثير قضايا مهنية وأخلاقية، لا سيما، تقويض الإبداع وغياب المراقبة والتحيز و الثفافية واستخدام البيانات.

وبالتركيز على الصحافة الآلية وتحليل المشكلات الأخلاقية والقانونية التي تواجه تلك النوعية من الصحافة المعتمدة على تقنيات الذكاء الاصطناعي جاءت دراسة (Monti, 2019) لإلقاء الضوء على حرية المعلومات و المسئولية ومدى تأثر تلك القضايا بالتكنولوجيا الجديدة واستخدام الذكاء الاصطناعي، وانتهى الباحث إلى أن القضية ليست في الكيفية التي يستخدم بها الصحفيون البرمجيات لتحقيق الصالح العام و انما القضية تتمثل في "كيف ومتى وأين ولماذا ومن" يتم استخدام تلك التكنولوجيا، ولتحقيق المسئولية و الثقة في تلك النوعية من الصحافة يلزم على المحررين أن يطوروا أدو اتهم في استكثاف الأخبار ومر اجعة البيانات من المصادر لتجنب التزييف و التضليل.

Wölker \& Powell, وعن الكيفية التي ينظر بها قراء الأخبار الأوربيون إلى الصحافة الآلية ومصداقيتها سعت دراسة (2018)، بعنوان البرمجيات (الخوارزميات) في غرف الأخبار: دراسة لإدراك قراء الأخبار لمصداقية الصحافة الآلية، وقد تم استخدام المنهج التجريبي في تلك الدراسة، إذ أجريت الدراسة على عينة من 300 مبحوث وتقيس الدراسة التجرييية رؤيتهم 
للصحافة الآلية من منظور الرسالة ومصداقية المصدر وتأثيرات ذللك على سلوكهم الانتقائي ومقارنتها بإدراكهم لمصداقية الصحافة القائمة على العمالة البشرية، وأظهرت الدراسة أن إدرالك المصداقية لكل من الصحافة الآلية و الصحافة القائمة على العمالة البشرية تكاد تكون منساوية، و إن كان قراء الأخبار يميلون إلى المضمون المنتج بواسطة آلة لعدم وجود تحييزات أو

$$
\text { ميل لطرف دون آخر. }
$$

وللتعرف على الكيفية التى يتم بها تطبيق التشغيل الآليAutomationفي غرف الأخبار النرويجية سعت الدراسة الأوربية حول استخدام الذكاء الاصطناعي في غرف الأخبار، فالصحافة الآلية تسمح بإنتاج أخبار ومحتوى (Pashevich, 2018) جديد ومبتكر، وقد قامت الدراسة على التحليل الكيفي للمقابلات المتعقة التي أجريت مع 11 صحفيا ومطوري النظم و المتخصصين العالمين في الصحافة الآلية بالنرويج و السويد و ألمانيا، وتشير الدراسة إلى أن جودة النص المنتج بالتكنولوجيا الجديدة مرضية ولكن لن تكون مميزة مثل المقالة المكتوبة يدويًا بو اسطة الصحفي. وعن استخدام الريبوتات في الصحافة تناولت الدراسة الأمريكية (Torrijos, 2019)، بعنوان التغطية الرياضية الآلية: دراسة حالة للريبوت المطلق بواسطة صحيفة واشنطن بوست خلال اولمبيات ريو 2016م وكوريا الجنوبية 2018م، وقد اختار الباحث دراسة حالة للمؤسسات الإعلامية التي تستخدم الذكاء الاصطناعي (صحيفة الو اشنطن بوست)، وتناولت كيف تطبق الصحافة الأمريكية الذكاء الاصطناعى في تغطيتها الإخبارية خلال أولومبيات صيف 2016م التي أقيمت في "ريو" وأولومبيات شتاء 2018م التي أقيمت في "كوريا الجنوبية"، ونم تحليل مضمون 999 رسالة إعلامية تم نشرها آلًَا في الصحيفة، وتوصلت الدراسة إلى أن استخدام تطبيقات الذكاء الاصطناعي يعمل على إرضاء المستخدمين الذين يحتاجون إلى أحدث الأخبار على كل أنواع الأجهزة التكنولوجية، ولم يكن هناك احتياج للتنخل البشرى في إنتاج الأخبار.

ومن الاراسات الأسبانية برزت دراسة(Tusa \&Tejedor, 2019)، بعنوان: "الأكاء الاصطناعي في الصحافة: حالة الصحفيين الرمزيين (الآليين) الرويوت والكمبيوتر (الحاسوبيين): دراسة من منظورالصحفيين"، هدفت هذه الدراسة إلى تحليل ظهور المقدمين الروبوتية والكمبيوترية باستخدام الذكاء الاصطناعي، واستخدمت الدراسة منهج تحليل المحتوى وذلك بتحليل الأخبار الموقعة من قبل الصحفيين في وسائل الإعلام الأمريكية والإسبانية، و التي يتم تصنيف معايير ها من خلال التقييم (لصالح وضد) استخدام الصحفيين الرمزيين (الآليين) في تجميع وتحرير الأخبار، وأوضحت نتائج الدراسة مدى التكامل الوظيفي الصحفي باستخدام التكنولوجيا؛ ومدى قرب العالم الصحفي من النهاية في مقابل استخدام آلية وتقنية وتكنولوجية الأعمال الصحفية، ومن ناحية أخرى أكدت الدراسة على فكرة الباحث بنروسيه (1991م)، والذي أطلق على الصحفيين الرمزبين (الآليين) اسم وكلاء التنفيذ. 
وجاءت الاراسة الأمريكية (Salazar, 2018)، بعنوان الريبوتات والأكاء الاصطناعي: تحديات جديدة للصحافة، تهدف الدراسة إلى تحليل تأثير تكنولوجيا الذكاء الاصطناعي والريبوتات في مجال الصحافة، والتعرف على مستقبل الصحافة و وتخصصيها في حال استخدام هذه التكنولوجيات، وقدمت الدراسة نماذج لهذا الاستخدام في نيويورك تايمز واستخدامها لتقنيات آلة التعلم للبحث عن نماذج للبيانات المالية لشركات الإعلام الخاصة به، واستخدمت الصحيفة الذكاء الاصطناعي لإنتاج و إدارة المضمون الصحفي، كذلك صحيفة لوس أنجلوس تايمز والتي استخدمت الذكاء الاصطناعي وسمته Bot Quake وبها تم نشر الأخبار الإلكترونية بدون تدخل بشري خاصة في أوقات الأزمات كوقوع الزلازل، استخدمت وكالة أسوشيتدبرس Automated insights و هي أداة للذكاء الاصطناعي تسمح بإنتاج نماذج من التقارير و التحليلات لدوري كرة السلة. وفي محساولة للتعرف على التحديات الأخـلاقيـة التي تواجـه صحسفة الخـوارزميـات أحـ أشكـال الذكـاء الاصطناعي في ضوء المجال الاجتماعي والمهني و التنظيمي سعت دراسة(Dörr \& Hollnbuchner, 2017) لتناول استخدام الخوارازميات "Algorithms" في إنتاج المحتوى، وركزت الدر اسة على مفهوم صحافة الخوارزميات والتي تقوم على استخدام البرمجيات في إنتاج المضمون الصحفي بدون الاستعانة بالصحفي، كذلك التعرف على طبيعة القضايا الأخلاقية المترتبة على استخدام تلك البرمجيات، وتقوم الدر اسة على تحليل للنظريات الأخلاقية لعلم الأخلاق و النفعية و الفضيلة التي من شأنها أن تفسر تلك الظاهرة و الاستحانة بتلك النظريات باعتبار ها أطر نظرية للار اسة، ويثير التحليل إلى وجود تحول ذي دلالة في المسئولية في إنتاج الأخبار، فمع ظهور هذا النوع من الصحافة أصبح الصحفي البشر ليس العامل الأخلافي الوحيد في عملية إنتاج الأخبار، بل أصبح هناك مشتركون في عملية الإنتاج مثل الوكالة التي تعمل باللغة المبرمجة والمؤسسات الإعلامية والمبرمج وجامعي البيانات.

\section{(ب) المحور الفرعى الثانى: اتجاهات القائمين بالاتصال نحو استخذام تقنيات الأكاء الاصطناعى بالمؤسسات الصحفية:-}

وللوقوف على العو امل المؤثرة في تقبل واستخدام القائمين بالاتصال لتقنيات الأكاء الاصطناعي استهدفت الاراسة العربية (بريك ،2020)، والتي تناولت رصد اتجاهات القائمين بالاتصال نحو استخدام تقنيات الذكاء الاصطناعي بالمؤسسات الصحفية في مصر والسعودية، وصولً لاتجاهاتهم نحو مستقبل استخدام تقنيات الذكاء الصناعي ، وذلك في إطار النظرية الموحدة لقبول واستخدام التكنولوجيا، اعتمد الباحث على منهج المسح ودر اسة العلاقات المتبادلة، وعينة كرة الثلج، وتم إجراء الدر اسة على عينة قو امها (143) مفردة ، وق توصلت الدراسة إلى أن مستوى استخدام هذه التقنيات جاء بشكل منخفض بنسبة 34.2\% يليها عدم استخدام تقنيات الذكاء الاصطناعي بنسبة 33.9\%، ثم بشكل متوسط بنسبة 29.5\%، و أخيرًا بشكل مرتفع بنسبة 5.6\%، كما توصلت الدر اسة إلى عدم وجود فروق ذات دلالة إحصائية بين اتجاه القائمين بالاتصال نحو استخدام تقنيات 
الذكاء الاصطناعي في المؤسسات الصحفية التي يعملون بها تبعًا للدولة التي تنتمي لها المؤسسة، كما جاء التصحيح التلقائي للأخطاء اللغوية أو المهنية في مقدمة تقنيات الذكاء الاصطناعي التي تعتمد عليها المؤسسات الصحفية المصرية والسعودية عينة الدر اسة بنسبة(89.5\%)، يليها إنتاج الأخبار بشكل آلي بنسبة(86.3\%). وفي محاولة لتفسير النية السلوكية كمؤشر لسلوك استخدام تلك التقنيات اعتمدت دراسة (الخولى، 2020)، على النظرية الموحدة لقبول واستخدام التكنولوجيا UTAUT في دراستها حول اتجاهات الصحفيين المصريين إزاء توظيف الذكاء الاصطناعي في تظويرالمضامين الصحفية الخاصة بالثراء المعلوماتي، والتي استهدفت الدراسة التعرف على اتجاهات الصحفيين المصربين إز اء توظيف تطبيقات الذكاء الاصطناعي في تطوير المضامين الصحفية الخاصة بالثراء المعلوماتي، كما اعتمدت الباحثة على منهج المسح باستخدام الاستبانة تم تطبيقها على عينة من الصحفيين العاملين بالمؤسسات المصرية قو امها 250 مفردة تم اختيار هم بطريقة عمدية من عدد (16) صحيفة مصرية، وتوصلت الاراسة إلى أن الصحف المصرية تعتمد على التقنيات الحديثة والتكنولوجية في العمل الصحفي في عمليات الجمع و التحرير و الإخر اج والنشر بدرجة كبيرة، كما أثتتت النتائج وجود علاقة ارتباطية ذات دلالة إحصائية بين اتجاهات الصحفيين المصريين نحو تطبيق تكنولوجيا الذكاء الاصطناعي في المؤسسات الصحفية ومتغيرات النظرية الموحدة لقبول التكنولوجيا ( توقع الأداء/ القلق من الاستخدام، المنفعة المتوقعة/ التأثيرات الاجتماعية/ التسهيلات المتاحة/ الجهد المتوقع).

وفي نفس السياق برزت دراسة (موسى، عبد الفتاح، 2020) عن اتجاهات الصحفيين والقيادات نحو توظيف تقنيات الذكاء الاصطناعي داخل غرف الأخبار بالمؤسسات الصحفية المصرية، حيث سعت الدر اسة إلى تحقيق هدفٍ رئيسى تمثل في الرصد الكمي و التفسير الكيفي لتصور ات ومو اقف الصحفيين العاملين في غرف الأخبار نحو توظيف تقنيات الذكاء الاصطناعي بغرف أخبار ها، وتحديد درجة جاهزيتها لتبني تلك التقنيات، و استندت الدر اسة في بناء متغير اتها وتفسير نتائجها على نظرية نشر الأفكار المستحدثة، ونموذج قبول التكنولوجيا، كما نم توظيف منهج المسح بشقيه الكمي والكيفي، وأداتي الاستبانة، و المقابلة المتعقة، على عينة قوامها 150 مفردة من الصحفيين المصريين العاملين بغرف الأخبار والقيادات الصحفية بالمؤسسات القومية والخاصة،، وتوصلت الدراسة إلى عدة نتائج أبرزها: أن نسبة 88 \% من إجمالي العينة من الصحفيين و القيادات يؤكدون على الأهمية الكبيرة لتوظيف تقنيات الذكاء الاصطناعي بغرف الأخبار الخاصة بهم، إلا أنهم أشاروا في نفس الوقت إلى عدم جاهزية نسبة كبيرة من غرف الأخبار لتوظيف هذه الأدوات نتيجة عدم تحديث الهياكل التنظيمية بها، بجانب عدم توفر خو ارزميات لتحرير النصوص بالنسخة العربية، ونر اجع الاستثمار والتمويل في هذه التقنية، و أظهرت النتائج 
أهم التاثير ات الإيجابية لتقنيات الذكاء الاصطناعي وتوظيفها في غرف الأخبار أنها توفر بيئة عمل أكثر راحة للصحفيين، وتعمل على تفر غهم للمهام الإبداعية.

وفي الإطار ذاته استهدفت دراسة (Broussard, Et al, 2019)، بعنوان اتجاهات الصحفيين نحو استخدام الأكاء

الاصطناعي في العمل الصحفي، التعرف على الدور الذي يقوم به الذكاء الاصطناعي في العمل الصحفي، والتعرف على الوظائف التي يمكن أن يقوم بها من وجهة نظر الصحفيين، وقد اعتمد الباحث على المنهج المسحي حيث قام باختبار مجموعة من الصحفيين قوامها 80 مفردة من الصحفيين مستعينًا باستمارة الاستقصاء، وقد أظهرت النتائج أنه على الرغم من قيام تكنولوجيا الذكاء الاصطناعي في معالجة البيانات و إظهار ها في شكل تقارير وأخبار صحفية إلا أن الأمر قد يتطلب في بعض الأحيان التذخل البشري في تعديل بعض الصياغات حتي يتسنى نشرها على الجمهور، كما أشنارت النتائج أن الذكاء الاصطناعي مكن من معالجة المعلومات والبيانات الصحفية في صورة ملائمة لأخلاقيات ومعايير ميثاق الشرف الصحفي. ومن منظور آخر أهتمت دراسة (Beckett, 2019)، بعنوان قوى ومسئوليات جديدة: دراسة مسحية عالمية حول الصحافة والأكاء الاصطناعي، بتناول مدى فهم الصحفيين لجوانب مفهوم الذكاء الاصطناعي وكيف يستخدمونه في غرف الأخبار وآرائهم حول المخاطر المحتملة من استخدام الذكاء الاصطناعي في صناعة الأخبار ، واعتمدت تللك الدراسة في جمع البيانات على أدوات المقابلات، فقد أجريت الدراسة على عينة من الصحفيين الذين يعملون في 71 من المؤسسات الإخبارية التي تستخدم الذكاء الاصطناعي وبلغ حجم العينة 116 صحفيًا، وكانت من أهم النتائج أن غالبية المبحوثين يرون أنفسهم خبراء تكنولوجيين وأنهم من فئة "المتبنيين الأوائل للرقمنة" وبالتالي لديهم وعي أكبر بالذكاء الاصطناعي من الصحفيين التقليديين، كما أظهرت الدراسة أن غرف الأخبار رغم أنها تبنت الذكاء الاصطناعي إلا أنه ينقصها الكثير من الإمكانات والأدوات

$$
\text { الضرورية لتطبيق الذكاء الاصطناعي بها. }
$$

في حين هدفت الدراسة الآسيوية (Kima \& Kim, 2018) بعنوان:"|تجاهات الصحفيين نحو تطبيق الأكاء الاصطناعي في العمل الصحفي"، إلى التعرف على اتجاهات الذكاء الاصطناعي في العمل الصحفي، اعتمد الباحث على المنهج المسحي، حيث قام باختيار عينة قوامها 47 صحفيًا من دولة كوريا الجنوبية، باستخدام استمارة الاستقصاء، أشارت نتائج الدراسة إلى أن اتجاهات الصحفيين نحو استخدام الذكاء الاصطناعي قدر العمل إلى ثلاث مجموعات المجموعة الأولى والتي نرى أن الإمكانات التي يتمتع بها الذكاء الاصطناعي أوصل الكثير من الصحف إلي مكانة الصحف الأولى أو النخبة الصحفية، أما المجمو عة الثانية وهي على النقيض من المجمو عة الأولى حتى أنه تم تصنيفها بأن لديهر عقدة فر اكنشتاين، حيث أظهرت تلك المجمو عة بعض المخاوف من استخدام ذلك الذكاء الاصطناعي مفسر اً ذلك على بعض التجارب السلبية في استخدام ذلك الذكاء 
الاصطناعي، مثل: الاختراق أو حدوث بعض الأعطال الحاسوبية التي تؤثر على جودة العمل الصحفي، أما المجموعة الثالثة و هي صاحبة وجهة النظر الوردية rosy view ، حيث إنها تقبل توظيف و استخدام الذكاء الاصطناعي وذلك لما يتمتع به من إيجابيات.

وتمثل التساؤل الرئيسي لاراسة (Galily, 2018)، حول اتجاه الصحفيين نحو استخدام الذكاء الاصطناعي في الصحافة الرياضية، ما هي اتجاهات الصحفيين نحو تلك التغيرات في العمل الصحفي؟ هل تعتبر بمثابة تطور للعمل الصحفي Sweeping change لنظام العمل الصحفي؟ اعنمد الباحث على المنهج المسحي حيث قام الباحث باختيار عينة من الصحفيين العاملين في المجال الرياضي قو امها 45 مفردة بحثية، مستعينًا باستمارة الاستقصاء، أشارت نتائج الدراسة إلى أن الصحفيين يرون أن استخدام الذكاء الاصطناعي يعتبر بمثالية تغيير شامل في العمل الصحفي نتيجة المهام المتعددة التي تقوم بها تلك التكنولوجيا، كذلك أشتارت اتجاهات الصحفيين أن استخدام التكنولوجيا والذكاء الاصطناعي قد سمح بوجود ما يعرف بإنتاج المحتوى الإلكتروني automated content production أو صحافة الروبوت gوrobo - journalism كما هدفت دراسة (شرقي، 2018) بعنوان أثز التكنولوجيا في إنتاج المعلومة في مضمون الصحافة المكتوبة"، إلى التعرف على اتجاهات الصحفيين نحو استخدام التكنولوجيا الحديثة في العمل الصحفي، اعتمدت الباحثة على المنهج المسحي، حيث قامت بدر اسة عينة من الصحفيين الجز ائريين قو امها 115 مفردة بحثية، أشارت اتجاهات الصحفيين أن تلك التكنولوجيا الحديثة أسهمت في سر عة توصيل الأخبار إلى القارئ بشكل منافس وسائل الإعلام الأخرى، كما أسهمت التكنولوجيا الحديثة من التقليل في التكلفة ومن تصحيح الأخطاء الإملائية والنحوية إلكترونيًا، كما أسهت نللك التكنولوجيا في الوصول إلى سيل مندفق ومتجدد من المعلومات والأخبار من مصادر متتو عة وفي شتى الموضوعات، قد يعز العنصر البشري عن القيام بها. وبالتركيز على مدى وعي الصحفيين بطبيعة تطبيقات الذكاء الاصطناعي كنظرية وكتطوير لأنظمة الحسب الآلي جاءت الدر اسة الأمريكية (Hansen, et al., 2017)، بعنوان: الأكاء الاصطناعي: الممارسة والتطبيقات بالنسبة للصحافة، وتدور الدراسة حول ما الذي ينبغي أن يعرفه الصحفيون عن تللك التكنولوجيات، وما الذي يجب أن تعرفه تللك التكنولوجيات عن المعايير الصحفية؟ وقد أجريت الدراسة بواسطة مركز Tow للصحافة الرقمية ومؤسسة Brown للمستحدثات الإعلامية خلال عام 2017م، وأظهرت النتائج أن برى المبحوثون أن الذكاء الاصطناعي قادر على تحسين مهمة الصحافة بدلاً من أن تحل محل الصحفيين، ويستحق القراء أن ينعرف على منهجية استخدام أدوات الذكاء الاصطناعي التي تستخدم في أداء تحليل ما أو التعرف على تقرير نتيجة ما في القصة الإخبارية. 
في حين أضافت الدراسة الآسيوية (Jung, et al., 2017)، حول إدخال الذكاء الاصطناعي في العمل الصحفي واتجاهات الصحفيين نحوها، وما هي تفضيلاتهم بين الاختيار بين العمل التقليدي أو توظيف الذكاء الاصطناعي، إضافة إلى التعرف على تفضيلات الجمهور بين المقالات المكتوبة بطريقة صناعية أو تللك التي يكتبها صحفيون فعليون، اعتمد الباحث على المنهج التجريبي حيث قام باختيار عينة مكونة من مجموعة من الصحفيين قو امها 35 صحفيًا وعينة أخرى من الجمهور قو امها 87 من الجمهور، وقد أثارت نتائج الدراسة أن الصحفيين يرون أهمية بالغة لتوظيف الذكاء الاصطناعي في العمل الصحفي نتيجة للسرعة التي يتمتع بها في معالجة الأخبار وفي بعض الأحيان تحليلها، كما أثنارت النتائج إلى أن الصحفيين بفضلون العمل الصحفي التقليدي وذللك في بعض القوالب الصحفية كالتحقيقات والتي تطلب النظر بين السطور وذللك لتحليل متغيرات يعجز عنها بعض التقنيات التكنولوجية، كما أكلت النتائج إلى عدم وجود اختلاف بين المقالات المكتوبة بطريقة صناعية أو تلك المكتوبة بطريقة تقليدية، لكن عندما يتعلق الأمر بمتابعة صحفي معين، فإن الجمهور في تلك الحالة ييحث عن كتابات ذلك الصحفي. المحور الثاني: استعراض الاراسات التي تناولت تأثيرالذكاء الاصطناعي في مجال التليفزيون:اقتصرت الدر اسات التي تم تناولها عبر هذا المحور و التي بلغ عددها (6) در اسات فقط جميعها من الدراسات الأجنبية ما بين (4) در اسات أوربية ودر استين أمريكيتين، ومن نماذج تلك الدراسات :-

برزت الدراسات الأوربية كدراسة (Ceide,et al., 2020)، بعنوان: تأثير كوفيد 19 على التليفزيون في أسبانيا واستخدام الروياتات: المحتويات، والمشاهدين، والدعم والاستراتيجيات للإنتاج، و استخدمت الدراسة منهجية مر اجعة البليو غر افيات الخاصة بتأثير COVID-19 في عملية التواصل وتفسير التقارير الواردة من الوكالات والمؤسسات ذات الصلة بتأثير إغلاق التلفزيون، وكثفت نتائج الدر اسة زيادة أعداد الجمهور المشاهد لثاشات التلفزيون التقليدية ومنصات البث المجانية لكل منهم، و أكدت نتائج الدراسة على ازدياد العرض والطلب على المحتوى الإعلامي، وعمل الروبوتات و الترفيه و العرض لجمهور الثباب، و أثر استخدام الرقمية والتقنية التكنولوجية في خدمات التلفزيون.

في حين آخر جاءت الدراسة الأمريكية (Hoyle \& Antelo, 2020)بعوان: مدى تأثير الأكاء الاصطناعي على البرامج التي يبثها تلفزيون جلوبو Globo TV: دراسة حالة، والتي هدفت إلى توضيح كيف يؤثر الذكاء الاصطناعي على إنثاء محتوى الوسائط المتعددة ومعالجته وتقديمه في البرامج التي يبثها تلفزيون جلوبو Globo TV، كما سعت الدر اسة إلى إظهار كيفية تأثر كل برنامج يتم بثه بتقنيات التعلم العميق، واعتمدت الدراسة على اختيار ثلاثة برامج إذاعية كأهداف لنشر خوارزميات الذكاء الاصطناعي في سير العمل بهدف زيادة الإنتاجية وتقليل التكلفة وابتكار طرق جديدة لإنشاء المحتوى، 
و أوضحت نتائج الدراسة أن تقنيات التعلم العميق التي يستخدمها تلفزيون جلوبولها فو ائد سواءً كانت على الإنتاجية أو الطرق الجديدة لإنشاء المحتوى، مما يتيح للذكاء الاصطناعي فرصًا جديدة لتحسين الإنتاجية في بيئة البث.

كذلك جاءت دراسة (Crespo-Pereira, et al., 2020) بعنوان: الأنظة الجديدة التقنية والتكنولوجية والحديثة لمعرفة مدى إيرردات التليفزيون: الأسس النظرية لتطوير نظام قائم على المعرفة، هدفت هذه الدراسة إلى التعرف علي الأنظمة الجديدة التقنية و التكنولوجية و الحديثة لمعرفة مدى إيرردات التليفزيون، و التعرف على الأسس النظرية لتطوير نظام قائم على المعرفة من أجل الوصول إلى استقرار القواعد والأنظمة الإبداعية، وتوصلت نتائج هذه الدراسة إلي أهمية التليفزيون التقني و التكنولوجي الذي يبث من خلال الإنترنت ومعرفة استخدامه تجارياً من خلال المنتجات، وأيضاً استخدامه من زاوية المجال الخدمي، ودلت النتائج عدم قدرة التليفزيون التقليدي على المنافسة مع تليفزيون الإنترنت كأداة للبث وباستخدامه لوسائل تقنية وتكنولوجية.

وأضافت دراسة (Crespo-Pereira, \& Legeren-Lago, 2019) بعنوان: استراتيجيات التليفزيون الأوروبي العام تجاه الطلب الإعلامي في العصر الرقمي القائم على الذكاء الاصطناعي: دراسة حالة التلفزيون البريطاني العام"، والتي تناولت فحص الاستر اتيجيات المختلفة في السياق والمحتوي الرقمي و التي لها علاقة بتوزيع المحتوى المميز والخدمي العام عبر جميع أنواع الثاثنات، وركزت هذه الدراسة على الصيغ المبتكرة الرئيسية التي يستخدمها التلفزيون البريطاني العام BBC لضمان جاذبية المعلومات وتقديم محتوى خدمة عامة في مواجهة المنافسة القوية التي تتوقعها إطار ات التلفزيونات الجديدة، واستخدمت الدراسة المنهج الوصفي وذللك من خلال تحليل هيئات البث العامة الاستراتيجيات الرئيسية في مجال خدمات المعلومات و المحتوى الترفيهي للتليفزيون الأوروبي بدر اسة حالة التليفزيون البريطاني، وأكدت الدراسة على استخدام برامج الروبوت للعمل من خلال منصات الرسائل ووسائل التواصل الاجتماعي مثل استخدام برنامج Messenger news bot، و أوضحت النتائج اعتماد الـ PSM على استراتيجيات مختلفة لزيادة وجودها في السياق الدولي وتوزيع الخدمة العامة والمحتوى المميز من خلال جميع أنواع الثناثـات وذللك على المستوى العالمي، كما أشارت نتائج الدر اسة إلى مدى تداعيات دمج الروبوتات على سير عل BBC، و الذي يسهل، من خلال تطبيق الويب Bot Builder ، عمل تحويل الأسئلة والأجوبة إلى محادثات طبيعية، ومن ناحية أخرى أكدت نتائج الدراسة على أهمية تخصيص المحتوى ورفع كفاعته في السرد القصصي و التصميم المرئي ل المعلومات. بينما تناولت دراسة (ITU, 2019) الأمريكية بعنوان: أنظمة الذكاء الاصطناعي لإنتاج البرامج وتبادلها، وقد هدفت هذه الدراسة إلى مناقثة وتقييم التطبيقات الحالية والجهود الجارية ذات الصلة ببرنامج البث على المدى القريب ومسار الإنتاج، 
حيث يجري إدخال تقنيات البث الجديدة التي يقودها الذكاء الاصطناعي إلى سير عمل البث، والغرض من هذه التقنيات هو زيادة الإنتاجية و الكفاءة والفرص الإبداعية أثناء إنتاج البر امج، إلى جانب نقل المعلومات إلى المشاهدين بسر عة ودقة وتلقائية، وقد اشتملت الدراسة على تصنيف التطبيقات ومجالات الفوائد التكنولوجية: تحسين سير العمل، وتحسين النطاق الترددي، و إنشاء المحتوى التلقائي، و إنشاء المحتوى من الأرشيفات القديمة، واختيار المحتوى لاستهداف تركيبات سكانية في الجمهور، وتحسين اختيار الأصول - إنشاء البيانات الوصفية، وتنسيب المنتج والإعلان الديناميكي للبث، وتخصيص المحتوى، وبهذه الطريقة، عملت الدر اسة على تسليط الضوء على المجالات التي تؤثر فيها أساليب خوارزمية ML / AI بالفعل على مسار كل من الإنشاء و العملية والتوزيع داخل بث البرنامج و إنتاجه،وتوصلت الدراسة إلى عدة نتائج أهمها تقديم وصفٍ للجهود البارزة الجارية لكل فئة من (الإنشاء و العملية والتوزيع داخل بث البرنامج و إنتاجه).

كما جاء عن استخدام التليفزيون الأوربي للذكاء الاصطناعي سعت در اسة(IvánPuentes,2018) بعنوان:"استخدام الإعلان للاكاء الاصطناعي كضمان لاستدامة التليفزيون في أوروبا في القطاعين العام والخاص، إلى معرفة تطور و اتجاهات وتحديات الإعلان التليفزيوني المستند على آلية الذكاء الاصطناعي، واستخدمت الدراسة المنهج الوصفي بشقه التحليلي، وكانت العينة هي الإعلانات الموجودة في كل من القطاعين السمعي والبصري في السوق الأوروبي، ونوصلت النتائج إلى مدى زيادة الإعلانات مع تطور الذكاء الاصطناعي، و أكدت الدراسة على زيادة الواقع الافتر اضي باستخدام الحوسبة الفائقة والتطوير الآلي و المتنامي.

القسم الثالث: عرض تحليلي ونقدي لبحوث ودراسات تأثير الأكاء الاصطناعي في مجالي الصحافة والتليفزيون:بعد عرض الدراسات التي تناولت الموضوعات ذات الاهتمام بموضوع بحوث تأثير الذكاء الاصطناعي في مجالي الصحافة و التليفزيون فقد خلصنا بعض الملاحظات النقدية التي يمكن أن نوجز ها في النقاط التالية:

\section{أولاً: فئة الموضوع أو القضية البحيّة والمجالات المرتبطة بها:}

\section{تطرقت الدراسات والبحوث عينة التحليل لعدة موضوعات وقضايا بحثية يمكن مناقشتها في النقاط التالية:}

- أوضحت الدر اسات التي تتاولت الصحافة والذكاء الاصطناعي تركيز الدراسات الأجنبية على تناول تأثثر استخدام الذكاء الاصطناعي في العمل الصحفي و على أداء المهام الصحفية وخاصة فيما يتعلق بأهية توظيف تقنيات الذكاء الاصطناعي في جمع ونشر المعلومات في غرف الأخبار، فضلاً عن إلقاء الضوء عن التحديات التي تعيق توظيف تللك التكنولوجيا، مروراً بمستقل صناعة الصحافة في ظل استخدام تقنيات الذكاء الصناعي، وذلك في مقابل قلة اهتمام الدراسات العربية 
- كما برز اهتمام المدرسة العربية بتناول اتجاهات القائمين بالاتصال نحو استخدام تقنيات الذكاء الاصطناعي بالمؤسسات الصحفية من خلال الوقوف على العو امل المؤثرة في تقبل واستخدام القائمين بالاتصال لهذه التقنيات ومعدلات استخدامهم لها، وصو لاً لاتجاهات القائمين بالاتصال نحو دور الذكاء الاصطناعي في تغيير الممارسة الصحفية، والوقـوف على الآثار المحتملة على مستقبل الصحفيين جراء توظيف تلك التطبيقات، ومقترحاتهم لتحقيـق الاستخدام الأمثل لها في مجال العمل الصحفي، مثل دراسات (بريك،2020)، (شرقى، 2018). على الرغم مما أشارت إليه نتائج الدراسة التحليلية من تفوق الدراسات الأجنبية من حيث عدد الأبحاث التي تناولت تأثثير الذكاء الاصطناعي في مجالي الصحافة والتليفزيون، والتي بلغ عددها (27) دراسة بنسبة بلغت(71.1\%)، في مقابل (11) دراسة عربية بنسبة (28.9\%)، إلا أن هذا الفارق يشير إلى تفوق حقيقي للمدارس الأجنبية خاصة الأوربية والأمريكية منها، فهو تفوق كمي ونوعي؛ حيث ركزت الدر اسات الأجنبية على تناول نأثثر الذكاء الاصطناعي في مجالي الصحافة و التليفزيون من خلال عدة زو ايا وتقديم إضافات موضو عية حقيقة، وتمثل ذلك بشكل واضح في الدر اسات التي تناولت تأثثير الذكاء الاصطناعي في مجال العمل الصحفي .

- وعلى مستوى تأثير الذكاء الاصطناعي في مجال التليفزيون أوضحت نتائج التحليل اهتمام محدود للمدرستين الأوربية والأمريكية التي تتاولت نأثثر الذكاء الاصطناعي في مجال التليفزيون والتي ركزت على تناول أثز استخدام الروبوتات و التكنولوجية الرقمية في خدمات التلفزيون وازدياد العرض والطلب على المحتوى الإعلامي، فضلاً عن تناول إدخال تقنيات البث الجديدة التي يقودها الذكاء الاصطناعي (AI) إلى سير عمل البث، وذلك لزيادة الإنتاجية والكفاءة وتقليل التكاليف وابتكار طرق جديدة لإنشاء المحتوى والفرص الإبداعية أثناء إنتاج البرامج، إلى جانب نقل المعلومات إلى المشاهدين بسر عة ودقة وتلقائية، مروراً بالكيفية التي يؤثر الذكاء الاصطناعي (AI) على إنشاء المحتوى ومعالجته من خلال الأتمتة وتقنيات التعلم العميق مثل در اسات(Hoyle \&Antelo,2020) (ITU,2019) )، وذلك في مقابل قصور لاى المدرستين العربية والأسيوية بتناول تللك الموضو عات. - أكلد نتائج التحليل على اهتمام المدارس الاجنبية مجتمعة (الأوروبية والأمريكيـة والآسيوية ) بلراسـة تطبيق تقتيـات الأكاء الاصطناعي في العمل الإعلامي في المجتمعات المختلفة مثل أمريكا وبريطانيا و الهند والصين و أسبانيا و إيطاليـا و ألمانيا و السويد و إيرلندا و النرويج وكوريا الجنوبية، ولم يكن هنـالك اهتمـام كبير مـن جانب الدر اسـات العربيـة بـإجر اء در اسات تتناول تللك الموضو عات في مجتمعات عربية فيما عدا بعض الدراسات العربية التي طبقت في(مصرو السـودية و الجز ائر ) على حد علم الباحثنان. 


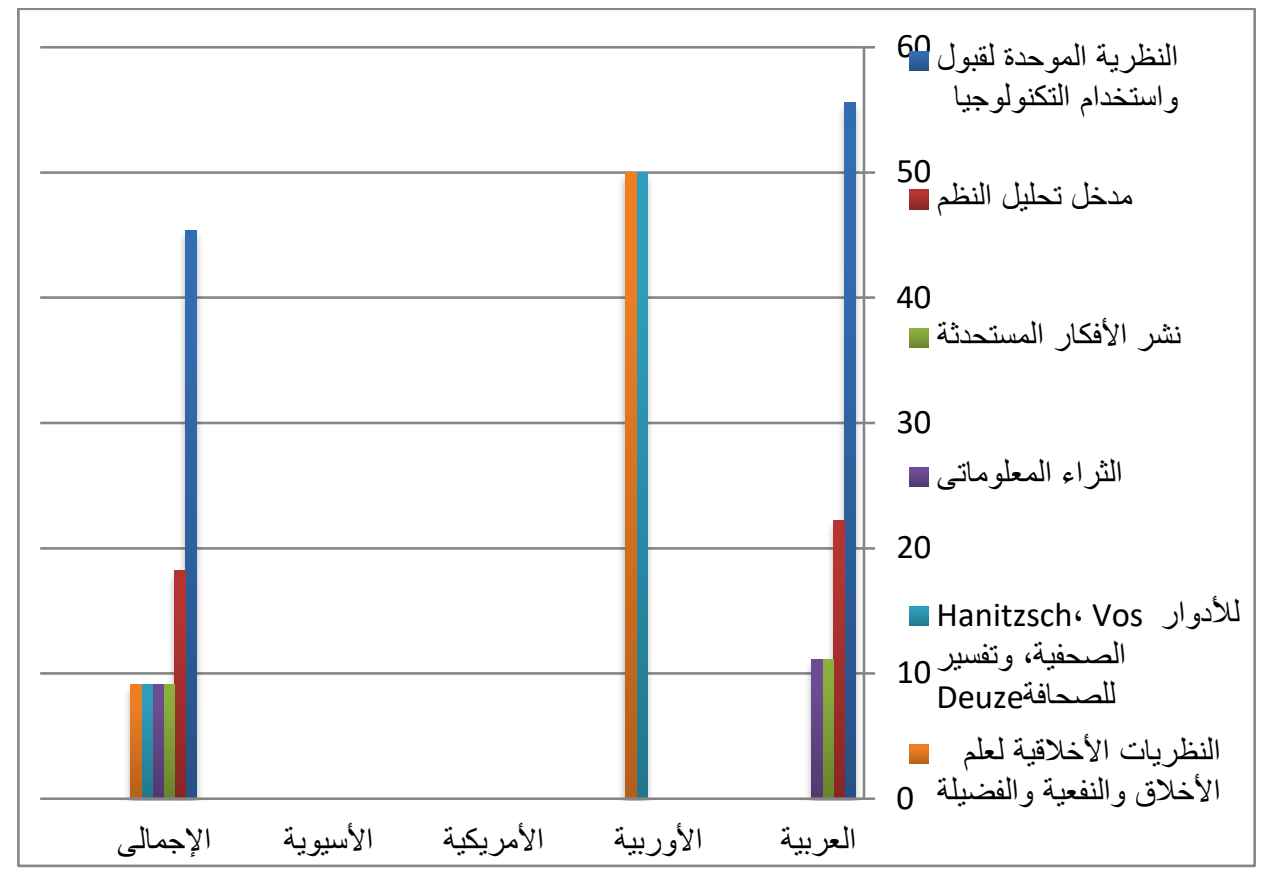

شكل(2) :يوضح الأطر النظرية المستخدمة في الاراسات والبحوث العربية والأجنبية عينة التحليل وفقاً للمدرسة البحثية

يتم في هذا الإطار استعراض الأطر النظرية المستخدمة للاراسات والبحوث العربية والأجنبية عينة التحليل كما يلي: - جاءت الدر اسات العربية في مقدمة الدراسات التي استندت إلى نماذج و أطر نظرية متتوعة و التي نم نوظيفها على المستوى التحليلي أو التفسيري حيث استخدمت (9) دراسة من (11) دراسة بنسبة (81.8\%) من الدراسات لنماذج و أطر نظرية، وذلك من إجمالي النظريات المستخدمة في الدراسات عينة التحليل، تليها الدراسات الأوربية بدر استين فقط بنسبة بلغت( 18.2\%) ، في حين غاب عن الدراسات الأمريكية والأسيوية استنادها إلى آطر نظرية محددة. - على الرغم من استناد دراسات بحوث الذكاء الاصطناعي وتأثيره في مجالي الصحافة و التليفزيون على مجموعة متنوعة من النظريات و النماذج و المداخل المهمة، والتي تم استخدامها على المستوى التحليلي أو التفسيري، إلا أن السيطرة جاعت للنظرية الموحدة لقبول واستخدام التكنولوجيا والتي تميزت بها الاراسات العربية بنسبة بلغت (45.4\%)، مثل در اسات (بريك،2020)، (الخولى، 2020)، (موسى عبد الفتاح، 2020)، (بدوى،2021) حيث حرص الباحثون على تقديمها لاختبار فروضها العلمية وتوظيفها لتفسير نتائج الدراسة، وتمثلت أبرز النظريات والمداخل التي استخدمتها الدراسات العربية عينة التحليل في مدخل تحليل النظم لدر استى(بدوى، 2020)،(منصور،2021) ،(نظرية الثراء المعلوماتي و التي استندت إليها الاراسة العربية (الخولى، 2020)، ودراسة (موسى عبد الفتاح، 2020) لنظرية نشر الأفكار المستحدثة. 
- بينما استندت الدراسات الأوربية كدراسة (Schapals \& Porlezza, 2020)لنموذج Vos ،Hanitzsch للأدوار الصحفية، وتفسير Deuze للصحافة، ودراسة (Dörr \& Hollnbuchner, 2017) للنظريات الأخلاقية لعلم الأخلاق و النفعية و الفضيلة، ولم يلاحظ استناد العديد من الدراسات الأجنبية عينة التحليل إلى أي من أطر نظرية محددة.

\section{يلاحظ من العرض التحليلى للاراسات العربية والأجنبية ما يلى :}

غاب عن معظم البحوث العربية التي استخدت إطار اً نظرياً تقديم الاتجاهات الجديدة للأطر النظرية التي نم استخدامها في موضو عات وبحوث تأثير الأكاء الاصطناعي في مجالي الصحافة والتليفيون. تبين أن النظرية الموحدة لقبول و استخدام التكنولوجيا كانت أعلى النظريات تطبيقاً. ثنالثاً : فئة التصميم المنهجى:يلاحظ من العرض التحليلي للمناهج والادوات المستخدمة في الاراسات العربية والأجنبية ما يلي : جدول(3): يوضح المناهج والأساليب البحثية المستخدمة في الاراسات عينة التحليل وفقاً للمدرسة البحثية

\begin{tabular}{|c|c|c|c|c|c|c|c|c|c|c|}
\hline \multicolumn{2}{|c|}{ الإجمالى } & \multicolumn{2}{|c|}{ الأسيوية } & \multicolumn{2}{|c|}{ الأمريكية } & \multicolumn{2}{|c|}{ الأوربية } & \multicolumn{2}{|c|}{ العربية } & \multirow{2}{*}{ 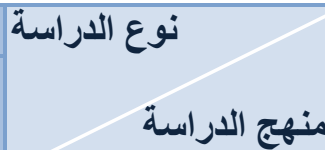 } \\
\hline$\%$ & ك & $\%$ & 5 & $\%$ & ك5 & $\%$ & 5 & $\%$ & ك5 & \\
\hline 31.6 & 12 & - & - & 33.3 & 4 & 33.3 & 4 & 33.3 & 4 & الكيفي \\
\hline 26.3 & 10 & 20 & 2 & - & - & 40 & 4 & 40 & 4 & المستح \\
\hline 23.7 & 9 & - & - & 11.1 & 1 & 66.7 & 6 & 22.2 & 2 & مختلط \\
\hline 7.9 & 3 & - & - & 66.7 & 2 & 33.3 & 1 & - & - & دراسة الحالة \\
\hline 5.3 & 2 & 50 & 1 & - & - & 50 & 1 & - & - & التجريب؟ى \\
\hline 5.3 & 2 & - & - & - & - & 50 & 1 & 50 & 1 & الوصفي \\
\hline 100 & 38 & 7.9 & 3 & 18.4 & 7 & 44.7 & 17 & 28.9 & 11 & الإجمالي \\
\hline
\end{tabular}

- - يتضح من الجدول السابق:- أن البحوث في المدرسة العربية التي عنيت بدراسة تأثير الذكاء الاصطناعي في مجالي الصحافة و التليفزيون قد اعتمدت على منهج المسح ، حيث سيطر استخدام منهج المسح الإعلامي على الدر اسات العربية والذي جاء في مقدة المناهج البحثية التي وظفتها تلك الدراسات عينة التحليل بنسبة بلغت(40\%)، لبحث المتغيرات المرتبطة بالموضو عات البحثية التي تثير ها تللك الدر اسات مثل دراسات (بلوى،2021)،(الخولى ،2020)،(موسى،عبد الفتاح ،2020)، (شرقى،2018) واستخدم بعض منها المنهج الكيفي بنسبة (33.3\%) كدر اسة (عبد المعطي،2020)،(نوفل،2020)،(أحمد،2018)،باستثناء دراستان عربيتان وظفت أكثر من منهج (مختلط) بنسبة بلغت(22.2\%) أحدهما دراسة (بريك،2020) وظفت كل من منهجي المسح بشقه الميداني ومنهج دراسة العلاقات المتبادلة، بينما وظفت الآخرى منهج المسح والمنهج المقارن وهي دراسة (بدوى،2020)، بينما اعتمدت

$$
\text { در اسة(منصور 2021)على المنهج الوصفي. }
$$


- بينما ركز المدرسة الأوربية على توظيف عدة مناهج حيث جاء الجمع بين أكثر من منهج (مختلط) بنسبة (66.7\%) كدراسة (Crespo-Pereira,et ( و التي جمعت بين المنهج الوصفي ومنهج المسح بشقه الميداني، ودر اسة (Schapals\&Porlezza,2020) al.,2020 وظفت منهجي الوصفي و المقارن، ودر اسة (Ufarte Ruiz\&Manfredi Sánchez,2019) والتي جمعت بين منهجي در اسة الحالة و المسح، وكذلك در اسة (Monti,2019) و التي وظفت المنهج الكيفي والاستقرائي، بالإضافة إلى دراسة (-Crespo(Pereira, \& Legeren-Lago, 2019 Ceide,et al., (المنهج المقارن والكيفي، كما وظفت المنهج الكيفي بنسبة بلغت(33.4\%) مثل دراسات، (Ali\&Hassoun,2019)(Dörr\&Hollnbuchner,2017)،(Tusa\&Tejedor,2019)،(2020 كما أستخدمت البعض منها منهج المسح وذلك بشقه الميداني كدر اسات (Broussard, Et al,2019)، دراسة (Beckett,2019)، دراسة (Galily,2018)، و المسح بشقه التحليلي مثل دراسة (Leavy,2020). ، ومنهج دراسة الحالة بنسبة(33.3\%) كدراسة (Braghieri,2019)، والمنهج التجريبي كدراسة (Wölker \& Powell,2018) ، والمنهج الوصفي التي وظفته دراسة واحدة فقط وهي .(IvánPuentes, 2018) - - مركزت المدرسة الأمريكية على توظيف كلا من المنهج الكيفي ودراسة الحالة، حيث اعتمدت النسبة الأكبر من تلك الدراسات على المنهج (20)(Tatalovic,2018)‘(ITU,2019)،(Stray,2019)(،\&Antelo,2020) في حين استخدت منهج دراسة الحالة في (2) در اسات فقط مثل در اسة (Torrijos,2019)، (Hansen,et al.,2017 ) ، في حين وظفت دراسة أمريكية واحدة منهجي الكيفي والتاريخي وهي (Salazar,2018).

على نحو آخر اعتمدت الدراسات الأسيوية على منهج المسح ووظفته داخل در استها كدراسة (Biswal \& Gouda, 2020) (Kung,et al.,2017)، في حين اعتمدت دراسة واحدة على المنهج التجريبي (Kima\& Kim,2018) 


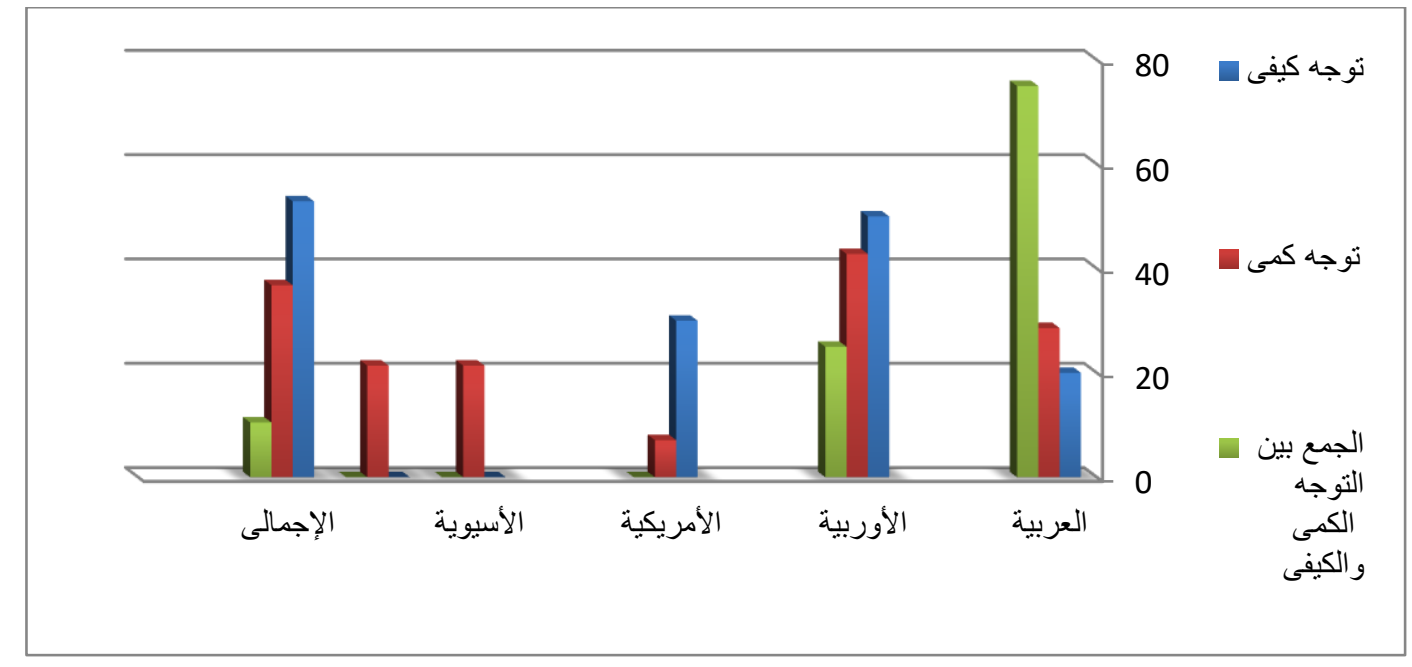

شكل(3): يوضح الاتجاه البحثي المستخدم في الاراسات والبحوث العربية والأجنبية عينة التحليل

- تنوعت الدراسات عينة التحليل من حيث المنـاهج البحثيـة حيث ظهرت العديــ مـن التصـيمات المنهجيـة حيث وظقت بعض الاراسات المنهج الكيفي واعتمدت بعضها على المنهج الكمي كما مزجت دراسات أخرى بين المنهجين الكمي والكيفي. • ويتضح من الثكل السابق بروز الاتجاه الكيفي في بحوث تأثثر الذكاء الاصطناعي في مجالي الصحافة والتليفزيون خلال فترة التحليل، في (20) در اسة بنسبة بلغت (52.6\%)، وظهر ذلك بشكل واضـح على مستوى الملارسـة الأوربيـة فظهرت (10) در اسة أوربية كيفية بنسبة (50\%)، في مقابل (6) در اسات أمريكية بنسبة بلغت (30\%)، بينمـا وجد (4) در اسـات عربيـة فقط بنسبة بلغت (20\%)، بينما لم يظهر التوجه الكيفي في أي من در اسات المدرسة الأسيوية. • بينما برز الاتجاه الكمي في (14) دراسة بنسبة بلغت (36.8\%)، خاصة في المدرستين الأوربية والعربيـة بعدد (6) در اسـات أوربية بنسبة بلغت(42.8\%)، في مقابل (4) در اسات عربيـة بنسبة بلغت (28.6\%)، وفي المدرسـة الأسيوية (3) در اسـات بنسبة (21.4\%)، بينما ظهر في در اسة واحدة أمريكية بنسبة بلغت (7.1\%). ثم جاء الجمع بين الاتجاه الكمي والكيفي في (4) در اسات عربية وأجنبية بنسبة بلغت (10.5\%) كان منها (3) دراسات عربية بنسبة بلغت(50\%)، و (1) دراسة عربية واحدة فقط، ودراسة أسيوية واحدة فقط، في حين لـم تظهر أي دراسـات أمريكيـة أو آسيوية تجمع بين التوجه الكمي والكيفي، ولكن يؤخذ على المدرسة العربية الكمية سيطرة طابع الجمود و التكرار، خصوصًا الدر اسات الميدانية العربية التي تناولت (اتجاهـات القـائمين بالاتصسال نحو استخدام تقنيـات الذذكاء الاصطناعي بالمؤسسـات الصحفية) فقد جاءت نتائجها متشابهة ومكررة، وأيضًا تشابه في العينات و الفئات التي طبقت عليها، ولم تقدم أي إضافات علمية، ولذلك نجد أن الدر اسـات العربيـة الخاصـة بالذكاء الاصطناعي وتـأثيره في مجـالي الصـحافة والتليفزيـون افتقدت إلى البعد التفسيري واكتفت بالبعد الوصفي فقط. 


\section{جدول(4):يوضح الأدوات الكمية والكيفية المستخدمة في الدراسات عينة التحليل وفقاً للمدرسة البحثية}

\begin{tabular}{|c|c|c|c|c|c|c|c|c|c|c|c|}
\hline \multicolumn{2}{|c|}{ الإجمالى } & \multicolumn{2}{|c|}{ الأسيوية } & \multicolumn{2}{|c|}{ الأمريكية } & \multicolumn{2}{|c|}{ الأوربية } & \multicolumn{2}{|c|}{ العربية } & \multirow[t]{2}{*}{ نوع الدراسة } & \multirow{2}{*}{ أدوت الدراسة } \\
\hline$\%$ & 5 & $\%$ & 5 & $\%$ & ك & $\%$ & ك & $\%$ & ك5 & & \\
\hline 21.1 & 8 & 12.5 & 1 & - & - & 37.5 & 3 & 50 & 4 & الإستبيان & أدوت كمية \\
\hline 5.3 & 2 & - & - & 50 & 1 & 50 & 1 & - & - & تحليل المضمون & \\
\hline 39.5 & 15 & - & - & 26.7 & 4 & 53.3 & 8 & 20 & 3 & تحليل المضمون & أدوات كيفية \\
\hline 10.5 & 4 & 25 & 1 & - & - & 50 & 2 & 25 & 1 & المقابلات المتعقةة & \\
\hline 2.6 & 1 & - & - & - & - & 100 & 1 & - & - & أكثرمن أداة كيفية & \\
\hline 2.6 & 1 & - & - & 100 & 1 & - & - & - & - & تالتاريخ الوثائق & \\
\hline 2.6 & 1 & - & - & 100 & 1 & - & - & - & - & $\begin{array}{r}\text { مجمو عات النقاش } \\
\text { المركزة }\end{array}$ & \\
\hline 5.3 & 2 & 50 & 1 & - & - & 50 & 1 & - & - & إختبار & أدوات عملية \\
\hline 2.6 & 1 & - & - & - & - & 100 & 1 & - & - & الاستبيان وتحليل الكيفي & الأدوات بين \\
\hline 5.3 & 2 & - & - & - & - & - & - & 100 & 2 & الاستبيان و المقابلات & والكيفية \\
\hline 2.6 & 1 & - & - & - & - & - & - & 100 & 1 & 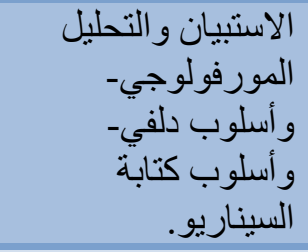 & \\
\hline 100 & 38 & 7.9 & 3 & 18.4 & 7 & 44.7 & 17 & 28.9 & 11 & & الإجمالي \\
\hline
\end{tabular}

كما يتضح من الجدول السابق: تنوعت الأدوات التي استخدمتها الدراسات الخاضعة للتحليل طبقا للمدرسة البحثية نتيجة لتتوع المناهج العلمية التي استخدمتها، فظهرت أدوات الدر اسة الكيفية إلى جانب الأدوات الكمية، فقد استعانت المدرسة الأوربية بعدد

Ceide,et من الأدوات الكيفية تصدر ها "تحليل المضمون الكيفي" بو اقع (8) در اسات بنسبة بلغت (53.3\%) مثل در اسات ‘(IvánPuentes,2018) (Dörr\&Hollnbuchner,2017) ‘(Monti,2019)‘ (Tusa \&Tejedor,2019) ‘al., 2020 كما حرصت المدرسة الأوربية على الجمع بين أكثر من أداة كيفية بواقع دراسة واحدة Ufarte Ruiz\&Manfredi) Sánchez,2019) كدر اسة (Peckett,2019)، (Pashevich, 2018)، ومن جاتب آخر ظهرت أدوات الدراسة الكمية تصدر ها الاستبيان بو اقع (3) در اسات منها (Broussard, Et al,2019)، (Braghieri, 2019)، (Galily,2018)، بينما أستعانت بتحليل المضمون الكمي دراسة (Leavy, 2020) ، كما جاءت دراسة آخرى استعانت بالاختبار كأداة عملية وهي \& Wölker) 
و Powell,2018) (Schapals\&Porlezza,2020) - ومن منظور أخر سيطرت الأدوات الكيفية على المدرسة الأمريكية حيث جاءت في مقدنها "تحليل المضمون الكيفي" بو اقع (4) در اسات كان منها (Hoyle \&Antelo,2020) (Stray,2019)،(Tatalovic,2018)، (ITU,2019) ، ثم جاءت كلا من جماعات المناقشة المركزة كدر اسة (Hansen, et al.,2017) و التحليل الوثائق التاريخية كدر اسة (Salazar, 2018)، Torrijos, ) بو اقع (1) در اسة واحدة لكل منهما، بينما استعانت دراسة واحدة أمريكية بتحليل المضمون الكمي وهي دراسة

- بينما نجد أن المدرسة العربية قد برزت في استخدام الاستبيان في 4 دراسات منها دراسة (بدوى،2021)،(بريك، 2020)؛(الخولى، 2020)، ودر اسة (شرقي، 2018) ، و استخدام تحليل المضمون الكيفي في 3 در اسات ، بينما لم تستخدم اياً من الأدوات الكيفية الآخرى كالمقابلات المتعقة أو مجموعات النقاش المركزة في آي دراسة، باسنثناء دراسة (عبد المعطى،2021) ، كما وجدت (3) دراسات جمعت بين الأدوات الكمية والكيفية كدراسة (بدوى ،2020) و التي استعانت بالاستبانة وأسلوب دلفي وكتابة السيناريو، وأيضاً دراسة (منصور،2021)، (موسى ،عبد الفتاح،2020) و التي وظفتا الاستبانة إلى جانب المقابلات المتعمقة.

- بينما اقتصرت المدرسة الأسيوية على استخدام أدوات القياس الكمي "الأستبيان" وظهر ذلك في در اسة واحدة وهي Kima (Biswal \& Gouda, \& Kim, 2018) ) (Jung,et al., 2017) في حين جاءت در اسة واحدة استعانت بالاختبار كأداة عملية وهي (2020)

\section{رابعاً: أهم النتائج البحثية التى خلصت اليها البحوث العربية والأجنبية:-}

ـ أوضحت نتائج الدراسات التي تناولت الصحافة والذكاء الاصطناعي تركيز المدرسة العربية والأوربية والأسيوية على تأثير استخدام الذكاء الإصطناعي في العمل الصحفي حيث أتفقت في مجملها على أن الذكاء الاصطناعي أسهم بشكل كبير فيما يتعلق بجمع وتحرير المعلومات، ومعالجة البيانات وتصحيح الأخطاء الإملائية والنحوية الذي يحدث إلكثرونيًا ودون التدخل المباثر من قبل المحريين ووضعها في شكل أخبار وتقارير صحفية قد منح السرعة والدقة في معالجة البيانات، مما سمح بسرعة نشر المعلومات إلى الجمهور، كما أن استخدام التكنولوجيا والذكاء الاصطناعي قد سمح بوجود ما يعرف بإنتاج المحتوى الإلكتروني Automated Content Production 
، الأمر الذي وفر الكثير من الوقت والجهد اللازم في العمل الصحفي، مثل دراسة (Biswal, \& Gouda, 2020) (بريك،Braghieri, 2019)، و(شرقي، 2020)(Galily,2018) . - - وأضافت نتائج المدارس الأوربية والأمريكية وتحديداً دراسة (Ufarte Ruiz\&Manfredi Sánchez,2019 و التى تتناولت در اسة حالة على شركة NarrativaInteligencia Artificial الأسبانية التي تكتب نصوصًا صحفية باستخدام برنامج يسمى Gabriele ، قد عمل على زيادة الإنتاجية الصحفية من خلال الإنتاج الآلي الدقيق والسريع للنصوص الصحفية والتي وصلت إلى 20 ألف نص في الأسبوع الواحد، وبتتبع النصوص التي تم إنتاجها لوحظ أنها لا تختلف كثير اً من حيث الأسلوب و التكوين و البناء عن الثكل التقليدي بالصحافة التقليدية، كما استخدمت وكالة أسوشيندبرس Automated insights وهي أداة للذكاء الاصطناعي تسمح بإنتاج نماذج من التقارير والتحليلات لدوري كرة السلة، كذلك صحيفة الواشنطن بوست استخدمت فeliograf bot أقيمت في"كوريا الجنوبية"، و هو ماأشارت إليه در استي (Torrijos,2019)، (Talazar,2018). ـ و أشارت نتائج در اسات المدرسة العربية إلى أهمية توظيف الروبوت في التحرير والتصوير، وفي تغطية الأحداث بالأماكن الأكثر خطورة ويصعب على الإنسان الوصول إليها مثل مناطق الحروب و الحر ائق وقاع البحار و الفضاء وفوق ناطحات السحاب، وكذللك استخدام تقنية الطائر ات بدون طيار(الدرونز) التي تتيح للصحفيين التقاط لقطات لأحداث إخبارية مثل الانفجار ات البركانية والقرى التي مزقتها الحروب و الكوارث الطبيعية، و هو ما أكدته الدر اسات العربية (عبد المعطى،2020)،(نوفل،2020)،(بدوى،2020). - ومن جاتب آخر أكدت نتائج دراسات المدرسة الأوربية(Ali \& Hassoun, 2019)، (Broussard, Et al, 2019)والأسيوية على أهمية تقنيات الذكاء الاصطناعي التي تمثل تطورًا كبيرًا في بيئة العمل الصحفي في العصر (Biswal, \& Gouda, 2020) الرقمي، خاصة في ظل قدرتها على التغلب على المشاكل الأساسية التي تواجه الصحافة المعاصرة ومكافحة الإشاعات والأخبار الز ائفة، وتحرير الأخبار وفقا لسياسة التحرير، كما أن الذكاء الإصطناعي مكن من معالجة المعلومات والبيانات الصحفية في صورة ملائمة لأخلاقيات ومعايير ميثاق الثرف الصحفي. ـ كما أوضحت نتائج الدراسات إلى عدم وجود اختلاف بين المقالات المكتوبة بطريقة صناعية أو تلك المكتوبة بطريقة تقليدية، لكن عندما يتعلق الأمر بمتابعة صحفي معين، فان الجهور في تللك الحالة يبحث عن كتابات ذلك الصحفي، وهو ما أثنارت إليه در اسات( (Jung,et al.,2017)،(Hansen,et al.,2017) (Wölker\& Powell,2018 جودة النص المنتج بالتكنولوجيا الجديدة ولكن لن يكون مميزًا مثل المقال المكتوب يدوياً بو اسطة الصحفي. 
- في حين اتفقت نتائج دراسات المدارس الأوربية والعربية والأسيوية في مجملها إلى أن الصحفيين برون أن استخدام الذكاء الاصطناعي يعتبر بمثابة نغيير شامل sweeping change في العمل الصحفي نتيجة المهام المنعدة والمتنوعة التي تقوم بها تلك التكنولوجيا في العمل الصحفي ،و أن غالبيتهم برون أنفسهم خبراء تكنولوجيين وأنهم من فئة "المتبنيين الأوائل للرقمنة" وبالتالي ل لديهم وعياً أكبر بالذكاء الإصطناعي من الصحفيين التقليديين مثل الددرسة الأوربية (Geckett, 2019)، (Galily, 2018) ) و أضاف بعضهم أن المستحدثات التكنولوجية لا تمثل لهم تهديداً ولا يقلقون عن احتمالية أن تحل الآلة محلهم في المستقبل وأن تلك رك التكنولوجيات هي أدوات مساعدة للصحفيين في آداء عملهم الأخباري اليومي مثل الدراسة العربية (الخولى،2020) ودراسة (موسى، عبد الفتاح ،2020)، والدر اسة الأوربية (Schapals \& Porlezza, 2020). ـ كما أظهرت نتائج دراسات الددرسة الأوربية والممثلة في دراسة (Beckett, 2019)، أنه من المهم أن تقوم المؤسسة بتعريف الذكاء الاصطناعي للمساعدة في رسم استر اتيجية محددة و الترويج لفهم الذكاء الاصطناعي و إدخاله في الغرف الاصطناعية، حيث إن غرف الأخبار رغم أنها تبنت الذكاء الاصطناعي إلا أنه ينقصها الكثير من الإمكانات والأدوات الضرورية لتطبيق الذكاء الاصطناعي بها، إضافة إلى ضرورة توفر بعض العناصر ذي تدريب وكفاءة عالية للتعامل مع تلك التطبيقات واستخدامها في الأعمال الصحفية. - و على نحو آخر أثنارت نتائج بعض دراسات الدارس العربية والأوربية والأمريكية والممثلة في دراسات تلك الددارس العلمية على النحو النالي، المدرسة العربية (موسى،عبد الفتاح،2020)، والأوربية(Ali\&Hassoun,2019) والأمريكية (Hansen,et) (al.,2017، أيضًا إلى أن استخدام تطبيقات الذكاء الاصطناعي في الصحافة يثير عددًا من القضايا مهنية و أخلاقية، لا سيما، تقويض الإبداع وغياب المراقبة والتحيز والثفافية والإنصاف واستخدام البيانات وجودة البيانات، وتصدر سلبيات الاستعانة بالذكاء الاصطناعي في العمل الصحفي السرقة المهنية والسطو على إنتاج الصحفيين، تلا ذلك تكاسل الصحفيين و عدم بذلهم الجهد الكافي في عملهم نتيجة الاستعانة بالتكنولوجيا الحديثة.

\section{يلاحظ من العرض التحليلى لنتائج الدراسات العربية والأجنية ما يلى:}

- طرحت صحافة الروبوت التي تعد أهم تقنيات الذكاء الاصطناعي الناتج عن التطور التكنولوجي في مجال الإعلام تحديداً جديداً أمام المؤسسات الصحفية في كيفية استخدمها ومدى تأثير ها على العملية الاتصالية بشكل كامل في المستقبل. - - إن للأكاء الاصطناعي دور في التعلم الآلي وأوضحت أن إنشاء المحتوى سيتم عن طريق الآلات وخوارزميات البرامج القادرة على تقليد قدرات الإدراك البشري. 
- - - إن الآلات الذكية لن تكون تهديدًا للبشر؛ على العكس من ذلك، سوف يتعامل معها البشر وينظرون إليها على أنها امتداد لهم للتكيف مع العالم الرقمي.

- - توقعت أن الذكاء الاصطناعى سيؤدي إلى طرق جديدة للتواصل واستخدام البيانات، وأن منصات التواصل الاجتماعي و التواصل الثخصي ستكون أكثر ذكاءً وتطورًا، وأن تطور وسائل الإعلام الجديدة هو أحد جوانب تقدم التكنولوجيا الذي لا يمكن إيقافه، و هو ما سيحدث تأثثرًا مباشرًا على كيفية استخدام البشر للبيانات ومشار كتها ونقلها.

- - إن الصحفيين يفضلون العمل الصحفي التقليدي وذلك في بعض القوالب الصحفية كالتحقيقات والتي تطلب النظر بين السطور وذللك لتحليل متغير ات و التي يعجز عنها بعض البرامج و التقنيات التكنولوجية. - - إن استخدام تطبيقات الذكاء الاصطناعي في الصحافة يثير عددًا من القضايا مهنية وأخلاقية، لا سيما، تقويض الإبداع و غياب المر اقبة و التحيز و الثفافية والإنصاف و استخدام البيانات وجودة البيانات.

القسم الرابع: الروية المستقبلية لبحوث الأكاء الاصطناعي وتأثيراته في مجال الصحافة والتليفزيون في ضوء القراعة النقدية لنتائج الدراسة التحليلية:-

\section{على مستوى الاتجاهات الخاصة بالموضوعات البحثية:}

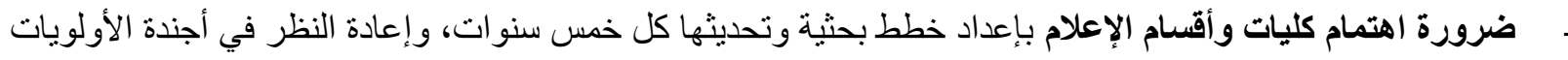
البحثية بحيث يتم توجيه الباحثين نحو الالنزام بتلك الخطط وتتاول موضوع نأثير الذكاء الاصطناعي على الإنتاج الإعلامي بما يتو افق مع المجالات التي أشتارت إليها الخطط البحثية. - تبرز أهميـة إجر اء در اسات عربية حول الاستخدام الأخلافي للاككاء الاصطناعي واحتر ام الممارسات الجيدة لاستخدام تلك التكنولوجيات الجديدة في مجال العمل الاعلامي. - ضرورة إجراء دراسـات تهتم بتقييم التطبيقات ذات الصلة ببرنامج البث ومسار الإنتاج، مـروراً بالكيفيـة التـي يـؤثر الذكاء الاصطناعي (AI) على إنشاء المحتوى ومعالجته وتقديمه في البر امج التـي يبثها التليفزيـون مـن خـلال الأتمتـة وتقنيـات التعلم العميق. - أهمية إجراء در اسات عربية حول تقييم القائمين بالاتصال لهذا الدور ورؤيتهم المستقبلية للارتقاء بالممارسة المهنية

$$
\text { و المعايير الأخلاقية عند نطبيق الذكاء الاصطناعي في العمل الإعلامي وخاصة الصحفي. }
$$

- أهمية تقديم در اسات لكيفية تعاون الإنسان والآلة في مجال العمل الإعلامي، و إعادة تعريف القائم بالاتصـال لنفسه وكيفية تطوير أدواته ليتو افق مع الوضع الجديد، واستخدام تلك التكنولوجيا. 
- أهمية إجراء دراسات عربية حول أهم المعوقات التي تحول دون الإفادة من تطبيقات الذكاء في المؤسسات الإعلامية العربية وخاصة المصرية، و إعداد الخطط للعاملين بهذا المجال للتمرن على الاستخدام الأفضل للبر امج المتطورة القائمة على الذكاء الاصطناعي و التعلم حول كيفية تطور تللك التكنولوجيا ومدى قدر اتها وإمكاناتها.

\section{على مستوى الاتجاهات الخاصة بأنماط البحوث العلمية:}

توجه الباحثين العرب نحو إجراء البحوث التجريبية لإيجاد نوع من التنوع في أنماط البحوث العربية على مستوى بحوث الذكاء الاصطناعي وتأثثيره في مجالي الصحافة و التلبفزيون.

\section{• • على مستوى الاتجاهات الخاصة بالأطر النظرية:}

- يقترح العرض التحليلي إجراء المزيد من الدراسات بالاعتماد على الأطر والنماذج النظرية الحديثة التي قدمها الباحثون وتوظيفها بالثكل الأمثل في بحوثهم سو اءً على المستوى التحليلي أو التفسيري. - ضرورة توجيه الباحثين لوضع تعريفات إجرائية لتحديد المفاهيم بدقة في بحوث الذكاء الاصطناعي ونأثيره في مجالي الصحافة والتليفزيون من خلال تأصيل مفهوم الذكاء الاصطناعى كنظرية وكتطوير لأنظمة الحاسب الآلي وكيفية استخدامه وتوظيفه في العمل الإعلامى.

\section{على مستوى الأطر المنهجية والأساليب البحثية المستخدمة:}

- ضرورة اهتمام المدرسة العربية بتنوع المناهج المستخدمة في در استها البحثية والاعتماد على أكثر من منهج، فضلاً عن أهمية التوجه نحو التنويع في استخدام الأدوات الكيفية على الدراسات العربية، مع ضرورة التكامل المنهجي باستخدام أكثر من أداة وخاصة في الدراسات الميدانية، بما يساعد على تفسير النتائج وتقديم رؤية واضحة حول تأثير الذكاء

$$
\text { الاصطناعي في مجالي الصحافة و التليفزيون. }
$$

- إن الدراسات العربية الخاصة بالذكاء الاصطناعي وتأثيره في مجالي الصحافة و التليفزيون افتقدت إلى البعد التفسيري و اكتقت بالبعد الوصفي فقط، ومن ثم ترى الباحثة أن ضرورة تركيز المدرسة العربية على استخدام الأدوات الكيفية كالمقابلات المتعمقة أوجماعات النقاش المركزة لتفسير النتائج بشكل أكثر دقة وعمق.

\section{على مستوى التدريس والتدريب:}

- ـ توصي الباحثة بضرورة إدراج مقرر دراسي يتناول الأكاء الاصطناعي وتأثيره في مجالي الصحافة والتليفيون ضمن المقررات الدراسية في جميع كليات و أقسام الإعلام بالجامعة العربية على أن بشمل المقرر الجانبين النظري والتطبيقي، ويضم الجزء التطبيقي دراسات حالة للمؤسسات الإعلامية في مجال الصحافة والتليفزيون التي تطبق أنظمة الذكاء 
الاصطناعي بها على جميع المستويات الدولية والعربية والمحلية، وفي نهاية المقرر يقدم الطالب على حسب تخصصه نموذجًا تجريبيًا لمنتج صحفي أو تليفزيوني باستخدام تطبيقات الذكاء الاصطناعي؛ حنى ينسنى لنا إخراج أجيال من الإعلاميين القادرين على استخدام التكنولوجيا الحديثة وتوظيفها بحرفية ومهنية عالية. ـ وبالنسبة للإعلاميين العاملين في المؤسسات الإعلامية تقترح الباحثة إعداد دورات تدريبية مكثفة لتدريب الصحفيين و إعادة تأهيلهم، و التمرن على الاستخدام الأمثل للبرامج المتطورة القائمة على الذكاء الاصطناعي، كذلك ضرورة تبني المؤسسات الصحفية اتجاهاً قوياً نحو توظيف صحافة الروبوت لمو اكبة التوجهات العالمية في سوق العمل الإعلامي. ـ في إطار التدفق التكنولوجي المستمر سيكون للثورة الرقمية تأثيرات سلبية، لذلك يوصي العرض التحليلي بضرورة الانتباه لخطورة تأثثر الذكاء الاصطناعي في مجالي الصحافة والتليفزيون بإجر اء مزيد من الأبحاث خاصة وأن التوقعات المستقبلية تؤكد استمر ار استخدامها والاعتماد عليها بشكل كبير . وفى ضوء الرويـة النقدية التى تم طرحها تقترح الباحثة قائمة بالموضوعات التىى بمكن تناولها فى بحوث الذكاء الاصطناعى وتأثيره فى مجالى الصحافة والتليفزيون، وذلك كما يلى:

- ـ ـ در اسات تتناول دور تقنية Heliograph في تطوير المحتوى الإلكتروني في مو اقع الصحف المصرية. - در اسات تجريبية عن أثر تقنية Narrative Science في كتابة المحتوى ومكافحة الأخبار الز ائفة عبر المنصات الرقمية. - دراسات تتناول أثر استخدام الروبوتات و التكنولوجيا الرقمية في إنتاج المحتوى وتوزيع البرامج، وآخرى عن أثر إدخال تقنيات البث الجديدة التي يقودها الذكاء الاصطناعي (AI) إلى سير عمل البث. - دراسات تتناول اتجاهات القائم بالاتصال نحو إنتاج المحتوى التليفزيوني باستخدام تقنيات الذكاء الاصطناعي، دراسات آخرى تتناول اتجاهات الجمهور نحو البر امج المنتجة بتقنيات الذكاء الاصطناعي. - دراسات تتناول المعايير المهنية والأخلاقية لصحافة الذكاء الاصطناعي، وأخرى عن مدى تفضيلات الجمهور لتلك النو عية من الصحافة. 


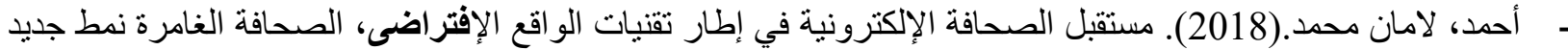

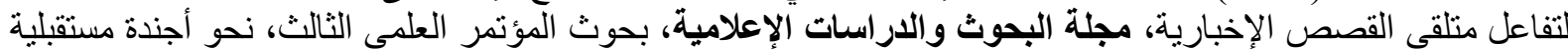

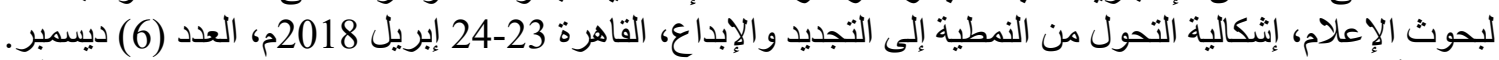

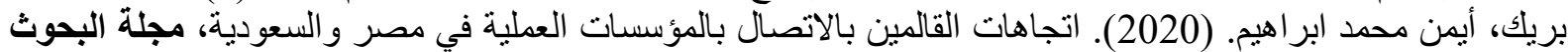

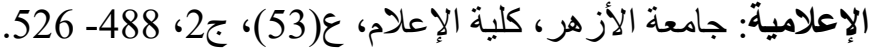

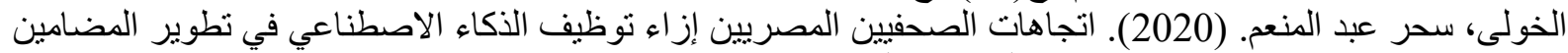

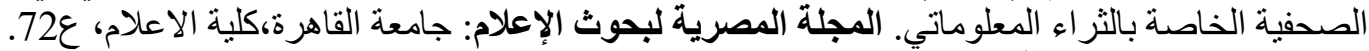

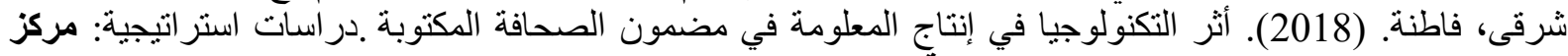

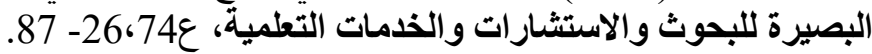

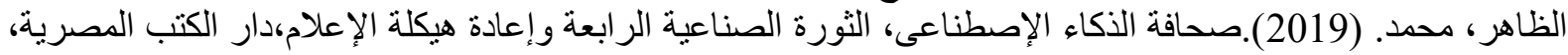

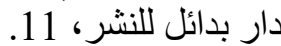

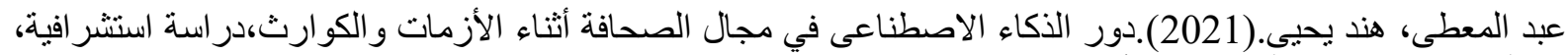

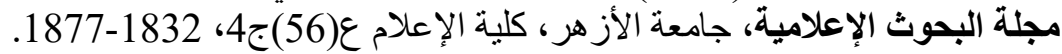

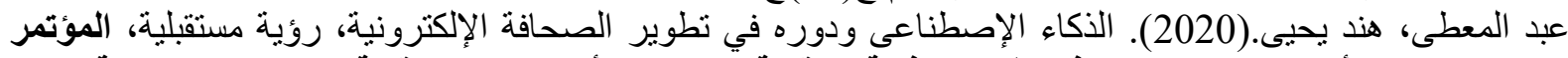

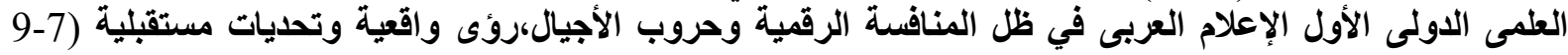

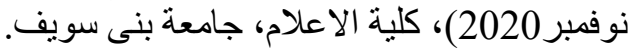

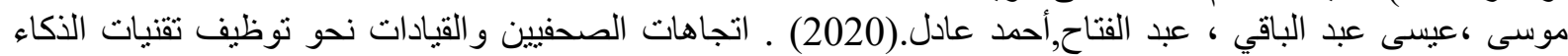

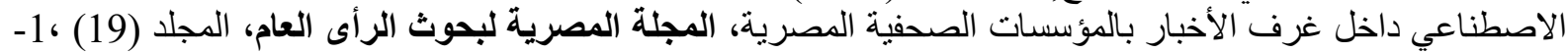

منصور، 67أحمد عبد المجيد.(2021) مستقبل الصحافة المصرية في ظل تقنيات الذكاء الاصطناعى خلال العقد من (2021-

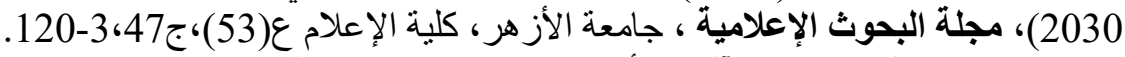

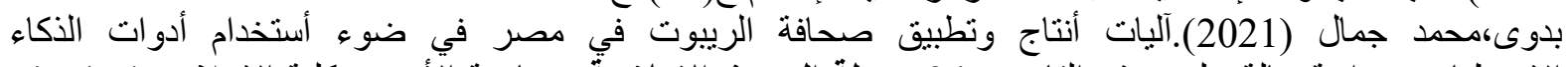
الاصطناعى،در اسة حالة على موقع القاهرى 242، مجلة البحوث الإعلاميةة ، جامعة الأزهر، كلية الإعلام ع(57)،47-

بدوى،محمد جمال.(2020). مستقبل الوظيفة الإخبارية للصحافة الإلكترونية في ظل تعدد منصات الإعلام الرقمي: دراسة

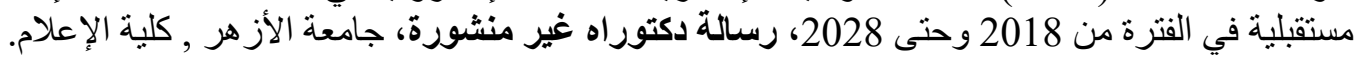

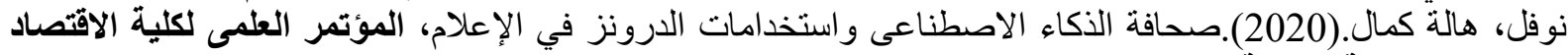
والعلوم السياسية بجامعة المستقبل، "مصر في عالم متغير".

- Ali, W., \&Hassoun, M. (2019). Artificial intelligence and automated journalism: contemporary challenges and new opportunities. Int J Media Journal Mass Commun, 5(1), $40-49$.

- Beckett, C. (2019). New powers, new responsibilities: A global survey of journalism and artificial intelligence. Polis, London School of Economics and Political Science. Https://Blogs. Lse. Ac. Uk/Polis/2019/11/18/New-Powers-New-Responsibilities.

- Biswal, S. K., \& Gouda, N. K. (2020). Artificial Intelligence in Journalism: A Boon or Bane? In Optimization in Machine Learning and Applications (pp. 155-167). Springer.

- Braghieri, M. (2019). Long-form journalism and archives in the digital landscape. King's College London.

- Broussard, M., Diakopoulos, N., Guzman, A. L., Abebe, R., Dupagne, M., \& Chuan, C.-H. (2019). Artificial intelligence and journalism. Journalism \& Mass Communication Quarterly, 96(3), 673-695.

- Ceide, C. F., López, M. T., \&Álvarez, M. V. (2020). Impact of the COVID-19 on television in Spain: Content, audience, platforms and production strategies. RISTI-RevistaIberica de Sistemas e Tecnologias de Informacao, 572-585. 
- Crespo-Pereira, Verónica; Legeren-Lago, B. (2019).European public television strategies in the face of the provision of information in the digital age: The case of the British public television",Journal of Information Systems and Technologies, E20,315-326. http://www.risti.xyz/issues/ristie20.pdf

- Dörr, K. N., \&Hollnbuchner, K. (2017). Ethical challenges of algorithmic journalism. Digital Journalism, 5(4), 404-419.

- Galily, Y. (2018). Artificial intelligence and sports journalism: Is it a sweeping change? Technology in Society, 54, 47-51.

- Hansen, M., Roca-Sales, M., Keegan, J. M., \& King, G. (2017). Artificial intelligence:

Practice and implications for journalism. 1-21.

https://academiccommons.columbia.edu/doi/10.7916/D8SN0NFD/download

- Hoyle, E., \&Antelo, A. (2020). How Artificial Intelligence impact the programs broadcasted by Globo TV: Case studies. SET INTERNATIONAL JOURNAL OF BROADCAST ENGINEERING, 5, 6. https://www.set.org.br/ijbe/ed5/artigo3.pdf

- International Telecommunication Union, \& ITU-R, R. E. P. O. R. T. (2019). Artificial intelligence systems for programme production and exchange". In Broadsiding Services (Television), ITU. Electronic Publication Geneva.

- IvánPuentes-Rivera. (2018). Advertising as a Sustainability Guarantee for European Private and Public Television". Journal RISTI, E16, 387-402.

- Jung, J., Song, H., Kim, Y., Im, H., \& Oh, S. (2017). Intrusion of software robots into journalism: The public's and journalists' perceptions of news written by algorithms and human journalists. Computers in Human Behavior, 71, 291-298.

- Kim, D., \& Kim, S. (2018). Newspaper journalists' attitudes towards robot journalism. Telematics and Informatics, 35(2), 340-357.

- Leavy, S. (2020). Uncovering Gender Bias in Media Coverage of Politicians with Machine Learning. ArXiv Preprint ArXiv:2005.07734.

- Monti, M. (2019). Automated journalism and freedom of information: ethical and juridi-cal problems related to AI in the press field. Retrieved from July 10, 2019.

- Pashevich, E. (2018). Automation of news production in Norway: Augmenting newsroom with artificial intelligence, University Of OSLO, Spring.

- Salazar, I. (2018). Robots and Artificial Intelligence. New challenges of journalism. DoxaComunicación, 27, 295-315.

- Schapals, A. K., \&Porlezza, C. (2020). Assistance or resistance? Evaluating the intersection of automated journalism and journalistic role conceptions. Media and Communication, 8(3), $16-26$.

- Stray, J. (2019). Making artificial intelligence work for investigative journalism. Digital Journalism, 7(8), 1076-1097.

- Tatalovic, M. (2018). AI writing bots are about to revolutionise science journalism: we must shape how this is done. SISSA Medialab.

- Torrijos, J. L. R. (2019). Automated sports coverages. Case study of bot released by The Washington Post during Río 2016 and Pyeongchang 2018 Olympics.

- Tusa, F., \&Tejedor, S. (2019).Artificial Intelligence in Journalism: the case of avatars and robotic presenters. A study from perception of journalist", Journal, RISTIU, E20, 267-279. 
- Ufarte Ruiz, M. J., \&Manfredi Sánchez, J. L. (2019). Algorithms and bots applied to journalism. The case of NarrativaInteligencia Artificial: structure, production and informative quality. DoxaComunicación, 29.

- Verónica Crespo Pereira, et. Others. "The new internet television income systems: theoretical foundation for the development of an Expert System based on knowledge", Journl, RISTI, N. ${ }^{\circ}$ E26, 02/2020,

Wölker, A., \& Powell, T. E. (2018). Algorithms in the newsroom? News readers' perceived credibility and selection of automated journalism. . Journalism, 